\title{
FRIEZES, STRINGS AND CLUSTER VARIABLES
}

\author{
IBRAHIM ASSEM \\ Université de Sherbrooke, Sherbrooke QC, Canada \\ E-mail: ibrahim.assem@usherbrooke.ca \\ GRÉGOIRE DUPONT \\ Université de Sherbrooke, Sherbrooke QC, Canada \\ E-mail: gregoire.dupont@usherbrooke.ca \\ RALF SCHIFFLER \\ University of Connecticut, Storrs CT, USA \\ E-mail: schiffler@math.uconn.edu \\ and DAVID SMITH \\ University Bishop's, Sherbrooke QC, Canada \\ E-mail:dsmith@ubishops.ca
}

(Received 14 September, 2010; revised 7 March, 2011; accepted 11 April, 2011; first published online 2 August 2011)

\begin{abstract}
To any walk in a quiver, we associate a Laurent polynomial. When the walk is the string of a string module over a 2-Calabi-Yau tilted algebra, we prove that this Laurent polynomial coincides with the corresponding cluster character of the string module up to an explicit normalising monomial factor.
\end{abstract}

2010 Mathematics Subject Classification. 13F60, 16G20.

0. Introduction. In the early 2000, Fomin and Zelevinsky [28] introduced the class of cluster algebras with the purpose of building a combinatorial framework for studying total positivity in algebraic groups and canonical bases in quantum groups. Since then, the study of cluster algebras was shown to be connected to several areas of mathematics, notably combinatorics, Lie theory, Poisson geometry, Teichmüller theory, mathematical physics and representation theory of algebras.

A cluster algebra is a commutative algebra generated by a set of variables, called cluster variables, obtained recursively by a combinatorial process known as mutation, starting from an initial set of cluster variables, the initial cluster, and a quiver without cycles of length at most two. One of the most remarkable facts about cluster variables is that they can be expressed as Laurent polynomials in terms of the initial cluster variables [28]; this is the so-called Laurent phenomenon. Also, it is conjectured that the coefficients in this expression are always non-negative; this is the positivity conjecture. The problem of computing explicitly the cluster variables is a difficult one and is the subject of wide literature. The most general results known at the present time are given in $[31,44,47]$.

In order to compute cluster variables, one may use friezes. Friezes, which go back to the works of Coxeter [21] and Coxeter-Conway [19, 20], are an efficient combinatorial tool that mimics the application of mutations on sinks or sources of the given quiver, 
hence an obvious combinatorial connection with cluster algebras $[\mathbf{4}, \mathbf{1 4}, \mathbf{1 7}, \mathbf{4 2}, \mathbf{4 8}]$. It is now known that connections between friezes and cluster algebras are deeper than just combinatorics, see for instance $[3,7,25,30,39,41]$. Our starting point for the present paper was the result given in [4] providing an explicit formula as a product of $2 \times 2$ matrices for all cluster variables in coefficient-free cluster algebras of type $\mathbb{A}$ and all but finitely many cluster variables in coefficient-free cluster algebras of type $\widetilde{\mathbb{A}}$, thus explaining at the same time the Laurent phenomenon and positivity. Our objective here is to show that the same technique can be used for computing the cluster variables associated with the string modules over a 2-Calabi-Yau tilted algebra (in the sense of [50]).

Besides friezes, our second main tool is the notion of a cluster character. In [14], Caldero and Chapoton noticed that cluster variables in simply laced coefficient-free cluster algebras of finite type can be expressed as generating series of the Euler-Poincaré characteristics of Grassmannians of submodules. Generalising this work, CalderoKeller [16], Palu [46] and Fu-Keller [31] introduced the notion of a cluster character associating to each module $M$ over a 2-Calabi-Yau tilted algebra $B_{T}$ a certain Laurent polynomial $X_{M}^{T}$ allowing one to compute a corresponding cluster variable (see also [47]). In general, cluster characters are hard to compute because one first needs to find the Euler characteristics of Grassmannians of submodules, and then the dimensions of certain Hom-spaces in the corresponding 2-Calabi-Yau category.

One class of algebras, however, whose representation theory is reasonably well understood is the class of string algebras, introduced by Butler and Ringel in [13] (see also [55]). In particular, indecomposable modules over string algebras are partitioned into two sets: string and band modules, and only string modules can be associated with cluster variables. The Euler characteristics of Grassmannians of submodules of string modules were computed by Cerulli and Haupt [18, 34]. Nevertheless, their methods do not allow one to compute explicitly the associated cluster character.

The main result of this paper gives an explicit formula for the cluster character associated with a string module over a 2-Calabi-Yau tilted algebra. This can be stated as follows. To any walk $c$ in a locally finite quiver $Q$, we associate a Laurent polynomial $L_{c}$ in the ring of Laurent polynomials in the indeterminates $x_{i}$ indexed by the set $Q_{0}$ of points of $Q$, which can be expressed as a product of $2 \times 2$ matrices as in [4] (see Section 1.3). Now, we let $T$ be a cluster tilting object in a Hom-finite triangulated 2-Calabi-Yau category $\mathcal{C}$ and $B_{T}=\operatorname{End}_{\mathcal{C}}(T)$ be the corresponding 2-Calabi-Yau tilted algebra whose ordinary quiver is denoted by $Q$. Moreover, to any string $B_{T}$-module $M$, we associate a tuple of integers $\mathbf{n}_{M}=\left(n_{i}\right)_{i \in Q_{0}}$, which we call the normalising vector of $M$ (see Section 5.2), and the Laurent polynomial $L_{M}$, which is just the Laurent polynomial $L_{c}$ attached to the string $c$ of $M$ in the quiver $Q$. Using the notation $\mathbf{x}^{\mathbf{n}_{M}}=\prod_{i \in Q_{0}} x_{i}^{n_{i}}$, our main result (Theorem 5.11) can be stated as saying that

$$
X_{M}^{T}=\frac{1}{\mathbf{x}^{\mathbf{n}_{M}}} L_{M}
$$

This result entails several interesting consequences. We first obtain the positivity of the Laurent polynomial $X_{M}^{T}$ for any string $B_{T}$-module $M$ (see Corollary 6.4), thus reproving the result of Cerulli and Haupt $[\mathbf{1 8}, \mathbf{3 4}]$. Our results also apply to the cases of string modules over cluster-tilted algebras, string modules over gentle algebras arising from unpunctured surfaces (see [2]) and, more generally, string modules over the finite dimensional Jacobian algebras associated with quivers with potentials in the sense of 
[22]. We also obtain a new proof of the positivity conjecture for cluster algebras arising from surfaces without punctures, see [51, 52].

The paper is organised as follows. Section 1 introduces the basic definitions and presents our combinatorial formula. Section 2 introduces the concept of realisable quadruples, which is the context in which our formula will actually compute cluster characters. This is closely related to the notion of triangulated 2-Calabi-Yau realisation in the sense of Fu and Keller [31]. Section 3 recalls all the necessary background from $[31,46]$ concerning cluster characters. Sections 4 and 5 contain the proof of our main result. In Section 4, we actually prove a weaker version of our theorem, which will be used in order to prove the general case in Section 5. In Section 6 we present possible applications of the results to the study of positivity in cluster algebras. In Section 7, we investigate the normalising factor and explicitly compute it for several cases of string modules over cluster-tilted algebras. The last section presents some detailed examples.

\section{The matrix formula.}

1.1. Notations. Throughout the article, $\mathbf{k}$ denotes an algebraically closed field. Given a quiver $Q$, we denote by $Q_{0}$ its set of points and by $Q_{1}$ its set of arrows. For any arrow $\alpha \in Q_{1}$, we denote by $s(\alpha)$ its source and by $t(\alpha)$ its target. We sometimes simply write $\alpha: s(\alpha) \rightarrow t(\alpha)$ or $s(\alpha) \stackrel{\alpha}{\rightarrow} t(\alpha)$. For any point $i \in Q_{0}$, we set

$$
Q_{1}(i,-)=\left\{\alpha \in Q_{1} \mid s(\alpha)=i\right\}, Q_{1}(-, i)=\left\{\alpha \in Q_{1} \mid t(\alpha)=i\right\}
$$

and if $F$ is a subset of points in $Q_{0}$, we set

$$
Q_{1}(i, F)=\left\{\alpha \in Q_{1} \mid s(\alpha)=i, t(\alpha) \in F\right\}, Q_{1}(F, i)=\left\{\alpha \in Q_{1} \mid s(\alpha) \in F, t(\alpha)=i\right\}
$$

and finally, for any $i, j \in Q_{0}$, we set $Q_{1}(i, j)=Q_{1}(i,-) \cap Q_{1}(-, j)$.

To any quiver $Q$, we associate a family $\mathbf{x}_{Q}=\left\{x_{i} \mid i \in Q_{0}\right\}$ of indeterminates over $\mathbb{Z}$. We set $\mathcal{L}\left(\mathbf{x}_{Q}\right)=\mathbb{Z}\left[x_{i}^{ \pm 1} \mid i \in Q_{0}\right]$ to be the ring of Laurent polynomials in variables $x_{i}$, with $i \in Q_{0}$ and $\mathcal{F}\left(\mathbf{x}_{Q}\right)=\mathbb{Q}\left(x_{i} \mid i \in Q_{0}\right)$ to be the field of rational functions in variables $x_{i}$, with $i \in Q_{0}$. For any $\mathbf{d}=\left(d_{i}\right)_{i \in Q_{0}} \in \mathbb{Z}^{Q_{0}}$, we set $\mathbf{x}_{Q}^{\mathbf{d}}=\prod_{i \in Q_{0}} x_{i}^{d_{i}}$.

A bound quiver is a pair $(Q, I)$ such that $Q$ is a finite quiver (that is $Q_{0}$ and $Q_{1}$ are finite sets) and $I$ is an admissible ideal in the path algebra $\mathbf{k} Q$ of $Q$. Given a finite dimensional $\mathbf{k}$-algebra $B$, there exists a bound quiver $(Q, I)$ such that $B \simeq \mathbf{k} Q / I$ and the quiver $Q$ is called the ordinary quiver of $B$ (see for instance, [5] or [6]). We always identify the category mod- $B$ of finitely generated right $B$-modules with the category $\operatorname{rep}(Q, I)$ of finite dimensional k-representations of $Q$ satisfying the relations in $I$. For a $B$-module $M$, we denote by $M(i)$ the $\mathbf{k}$-vector space at the point $i \in Q_{0}$ and $M(\alpha)$ the k-linear map at the arrow $\alpha \in Q_{1}$.

1.2. Walks and strings. Let $Q$ be a quiver. As in [13], for any arrow $\beta \in Q_{1}$, we denote by $\beta^{-1}$ a formal inverse for $\beta$, with $s\left(\beta^{-1}\right)=t(\beta), t\left(\beta^{-1}\right)=s(\beta)$ and we set $\left(\beta^{-1}\right)^{-1}=\beta$.

A walk of length $n \geq 1$ in $Q$ is a sequence $c=c_{1} \cdots c_{n}$, where each $c_{i}$ is an arrow or a formal inverse of an arrow such that $t\left(c_{i}\right)=s\left(c_{i+1}\right)$ for any $i \in\{1, \ldots, n-1\}$. The source of the walk $c$ is $s(c)=s\left(c_{1}\right)$ and the target of the walk $c$ is $t(c)=t\left(c_{n}\right)$. We define 
a walk $e_{i}$ of length zero for any point $i \in Q_{0}$ such that $s\left(e_{i}\right)=t\left(e_{i}\right)=i$. For any walk $c$, we denote by $c_{0}$ the walk of length zero $c_{0}=e_{s(c)}$.

If $(Q, I)$ is a bound quiver, a string in $(Q, I)$ is either a walk of length zero or a walk $c=c_{1} \cdots c_{n}$ of length $n \geq 1$ such that $c_{i} \neq c_{i+1}^{-1}$ for any $i \in\{1, \ldots, n-1\}$ and neither walk of the form $c_{i} c_{i+1} \cdots c_{t}$ nor its inverse belongs to $I$ for $1 \leq i$ and $t \leq n$. If $Q$ is a quiver, a string in the quiver $Q$ is a string in the bound quiver $(Q,(0))$. If $B \simeq \mathbf{k} Q / I$ is a finite dimensional k-algebra and $c$ is a string in $(Q, I)$, then we also say that $c$ is $a$ string in $B$.

To any string in a finite dimensional $\mathbf{k}$-algebra $B$ with bound quiver $(Q, I)$, we can naturally associate an indecomposable representation $M_{c}$ of the quiver $Q$. If $M_{c}$ is a representation of the bound quiver $(Q, I)$, then it determines a $B$-module $M_{c}$, which we call a string module as in [13, Section 3]. More precisely, we define $M_{c}$ as follows. If $c=e_{v}$ has length zero, then $M_{c}$ is the simple representation $S_{v}$ at point $v \in Q_{0}$. Otherwise, $c$ is a string of length $n \geq 1$ and we write $c=v_{1} \frac{c_{1}}{c_{n}} v_{n+1}$. For any $v \in Q_{0}$, we set $I_{v}=\left\{i \in[1, n+1] \mid v_{i}=v\right\}$ and define $M_{c}(v)$ as the $\left|I_{v}\right|$-dimensional $\mathbf{k}$-vector space with basis $z_{i}$, with $i \in I_{v}$. For any $1 \leq i \leq n$, if $c_{i}=\beta \in Q_{1}$, we set $M_{c}(\beta)\left(z_{i-1}\right)=z_{i}$ and if $c_{i}=\beta \in Q_{1}^{-1}$, we set $M_{c}(\beta)\left(z_{i}\right)=z_{i-1}$. Finally, if $\alpha: v \longrightarrow v^{\prime}$ is an arrow in $Q_{1}$ and if $z_{j}$ is one of the basis vector of $M_{c}(v)$ such that $M_{c}(\alpha)\left(z_{j}\right)$ is not yet defined, we set $M_{c}(\alpha)\left(z_{j}\right)=0$.

A string module is also called a string representation of the corresponding bound quiver. For any string module $M$, we denote by $\mathbf{s}(M)$ the corresponding string.

1.3. A formula for walks. For any locally finite quiver $Q$, we define a family of matrices with coefficients in $\mathbb{Z}\left[\mathbf{x}_{Q}\right]=\mathbb{Z}\left[x_{i} \mid i \in Q_{0}\right]$ as follows:

For any arrow $\beta \in Q_{1}$, we set

$$
A(\beta)=\left[\begin{array}{cc}
x_{t(\beta)} & 0 \\
1 & x_{s(\beta)}
\end{array}\right] \text { and } A\left(\beta^{-1}\right)=\left[\begin{array}{cc}
x_{t(\beta)} & 1 \\
0 & x_{s(\beta)}
\end{array}\right] \text {. }
$$

Let $c=c_{1} \cdots c_{n}$ be a walk of length $n \geq 1$ in $Q$. For any $i \in\{0, \ldots, n\}$ we set

$$
v_{i+1}=t\left(c_{i}\right)
$$

(still with the notation $c_{0}=e_{s(c)}$ ) and

$$
V_{c}(i)=\left[\begin{array}{cc}
\prod_{\substack{\alpha \in Q_{1}\left(v_{i},-\right) \\
\alpha \neq c_{i}^{ \pm 1}, c_{i-1}^{ \pm 1}}} x_{t(\alpha)} & 0 \\
0 & \prod_{\substack{\alpha \in Q_{1}\left(-, v_{i}\right) \\
\alpha \neq c_{i}^{ \pm 1}, c_{i-1}^{ \pm 1}}} x_{s(\alpha)}
\end{array}\right] .
$$

We then set

$$
L_{c}=\frac{1}{x_{v_{1}} \cdots x_{v_{n+1}}}\left[\begin{array}{ll}
1, & 1
\end{array}\right] V_{c}(1)\left(\prod_{i=1}^{n} A\left(c_{i}\right) V_{c}(i+1)\right)\left[\begin{array}{l}
1 \\
1
\end{array}\right] \in \mathcal{L}\left(\mathbf{x}_{Q}\right)
$$


If $c=e_{i}$ is a walk of length 0 at point $i$, we similarly set

$$
V_{e_{i}}(1)=\left[\begin{array}{cc}
\prod_{\alpha \in Q_{1}(i,-)} x_{t(\alpha)} & 0 \\
0 & \prod_{\alpha \in Q_{1}(-, i)} x_{s(\alpha)}
\end{array}\right],
$$

and

$$
L_{e_{i}}=\frac{1}{x_{i}}[1, \quad 1] V_{e_{i}}(1)\left[\begin{array}{l}
1 \\
1
\end{array}\right] \in \mathcal{L}\left(\mathbf{x}_{Q}\right) .
$$

In other words, if $c$ is any walk, either of length zero or of the form $c=c_{1} \cdots c_{n}$, we have

$$
L_{c}=\frac{1}{\prod_{i=0}^{n} x_{t\left(c_{i}\right)}}\left[\begin{array}{ll}
1, & 1
\end{array}\right]\left(\prod_{i=0}^{n} A\left(c_{i}\right) V_{c}(i+1)\right)\left[\begin{array}{l}
1 \\
1
\end{array}\right] \in \mathcal{L}\left(\mathbf{x}_{Q}\right)
$$

with the convention that $A\left(c_{0}\right)$ is an identity matrix.

EXAMPLE 1.1. Consider the quiver

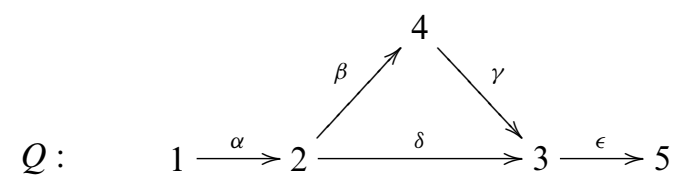

and consider the path $c=\delta^{-1} \beta \gamma$ in $Q$. Then

$$
\begin{aligned}
L_{c}= & \frac{1}{x_{2} x_{3}^{2} x_{4}}\left([1,1]\left[\begin{array}{cc}
x_{5} & 0 \\
0 & x_{4}
\end{array}\right]\left[\begin{array}{cc}
x_{3} & 1 \\
0 & x_{2}
\end{array}\right]\left[\begin{array}{cc}
1 & 0 \\
0 & x_{1}
\end{array}\right]\right. \\
& \left.\times\left[\begin{array}{cc}
x_{4} & 0 \\
1 & x_{2}
\end{array}\right]\left[\begin{array}{cc}
1 & 0 \\
0 & 1
\end{array}\right]\left[\begin{array}{cc}
x_{3} & 0 \\
1 & x_{4}
\end{array}\right]\left[\begin{array}{cc}
x_{5} & 0 \\
0 & x_{2}
\end{array}\right]\left[\begin{array}{l}
1 \\
1
\end{array}\right]\right) \\
= & \frac{x_{1} x_{2}^{3} x_{4}^{2}+2 x_{1} x_{2}^{2} x_{4} x_{5}+x_{1} x_{2} x_{3} x_{4} x_{5}+x_{3}^{2} x_{4} x_{5}^{2}+x_{1} x_{2} x_{5}^{2}+x_{1} x_{3} x_{5}^{2}}{x_{2} x_{3}^{2} x_{4}} .
\end{aligned}
$$

\subsection{A formula for string modules.}

DEFINITION 1.2. Let $B$ be a finite dimensional k-algebra with bound quiver $(Q, I)$ and $M$ be a string $B$-module with corresponding string $\mathbf{s}(M)$. We set

$$
L_{M}=L_{\mathbf{s}(M)} \in \mathcal{L}\left(\mathbf{x}_{Q}\right) .
$$

If $Q$ is a quiver, a subquiver $R$ of $Q$ is a quiver $R$ such that $R_{0} \subset Q_{0}$ and for any $i, j \in R_{0}$, the set of arrows from $i$ to $j$ in $R_{1}$ is a subset of the set of arrows from $i$ to $j$ in $Q_{1}$. If $R$ is a subquiver of $Q$, we naturally identify $\mathcal{L}\left(\mathbf{x}_{R}\right)$ with a subring of $\mathcal{L}\left(\mathbf{x}_{Q}\right)$.

A subquiver $R$ of $Q$ is called a full subquiver if for all $i, j \in R_{0}$ the set of arrows from $i$ to $j$ in $R_{1}$ equals the set of arrows from $i$ to $j$ in $Q_{1}$. If $B$ is a finite dimensional 
k-algebra with bound quiver $(Q, I)$ and $M$ is a $B$-module, the support of $M$ is the full subquiver $\operatorname{supp}(M)$ of $Q$ consisting of the points $i \in Q_{0}$ such that $M(i) \neq 0$. The closure of the support of $M$ is the full subquiver $\overline{\operatorname{supp}(M)}$ of $Q$ formed by the points $i \in Q_{0}$, which are in the support of $M$ or such that there exists an arrow $\alpha$ such that $s(\alpha) \in \operatorname{supp}(M)_{0}$ and $t(\alpha)=i$ or $t(\alpha) \in \operatorname{supp}(M)_{0}$ and $s(\alpha)=i$.

With these identifications, if $B$ is a finite dimensional k-algebra and $M$ is a string $B$-module, then

$$
L_{M} \in \mathcal{L}\left(\mathbf{x}_{\overline{\operatorname{supp}(M)}}\right)
$$

2. Realisable quadruples. In the previous section we associated with any string module $M$ over a finite dimensional algebra $B$ a certain Laurent polynomial $L_{M}$. In this section we provide a context in which the algebra $B$ arises in connection with some cluster algebras so that we can compare the Laurent polynomials $L_{M}$ with cluster variables or, more generally, with cluster characters. The context in which we work is the context of triangulated 2-Calabi-Yau realisations introduced in [31].

2.1. Definitions. An ice quiver is a pair $(\mathcal{Q}, F)$ such that $\mathcal{Q}$ is a finite connected quiver without loops and 2-cycles and $F$ is a (possibly empty) subset of points of $\mathcal{Q}_{0}$, called frozen points, such that there are no arrows between points in $F$. The unfrozen part of $(\mathcal{Q}, F)$ is the full subquiver of $\mathcal{Q}$ obtained by deleting the points in $F$.

For any quiver $Q$, we denote by $B(Q)=\left(b_{i j}\right) \in M_{Q_{0}}(\mathbb{Z})$ the skew-symmetric matrix defined for any $i, j \in Q_{0}$ by

$$
b_{i j}=\left|Q_{1}(i, j)\right|-\left|Q_{1}(j, i)\right| .
$$

If $(\mathcal{Q}, F)$ is an ice quiver with unfrozen part $Q$, then we can fix an ordering of the points in $\mathcal{Q}_{0}$ such that

$$
B(\mathcal{Q})=\left[\begin{array}{cc}
B(Q) & -C^{t} \\
C & 0_{F \times F}
\end{array}\right],
$$

where $C$ is a matrix in $M_{F \times Q_{0}}(\mathbb{Z})$.

For any ice quiver $(\mathcal{Q}, F)$ with unfrozen part $Q$, we denote by $\mathcal{A}(\mathcal{Q}, F)$ the cluster algebra of geometric type with initial seed $(\widetilde{B}(\mathcal{Q}), \mathbf{x}, \mathbf{y})$, where

$$
\widetilde{B}(\mathcal{Q})=\left[\begin{array}{c}
B(Q) \\
C
\end{array}\right],
$$

$\mathbf{x}=\left(x_{i}, i \in Q_{0}\right)$ and $\mathbf{y}=\left(x_{i}, i \in F\right)$ (see [29]).

A k-linear category $\mathcal{C}$ is called $\operatorname{Hom}$-finite if $\operatorname{Hom}_{\mathcal{C}}(M, N)$ is a finite dimensional k-vector space for any two objects $M$ and $N$ in $\mathcal{C}$. A k-linear triangulated category $\mathcal{C}$ is called 2-Calabi-Yau if there is a bifunctorial isomorphism

$$
\operatorname{Hom}_{\mathcal{C}}(X, Y) \simeq D \operatorname{Hom}_{\mathcal{C}}(Y, X[2])
$$

where $D=\operatorname{Hom}_{\mathbf{k}}(-, \mathbf{k})$ is the standard duality and [1] denotes the suspension functor. 
For any objects $M$ and $N$ in $\mathcal{C}$, we denote by $\operatorname{Ext}_{\mathcal{C}}^{1}(M, N)$ the $\operatorname{space}_{\mathcal{C}} \operatorname{Hom}_{\mathcal{C}}(M, N[1])$. An object $M$ in the category $\mathcal{C}$ is called rigid if $\operatorname{Ext}_{\mathcal{C}}^{1}(M, M)=0$. An object $T$ in $\mathcal{C}$ is called a cluster-tilting object in $\mathcal{C}$ if it is maximal and rigid, that is if for any object $X$ in $\mathcal{C}$, the equality $\operatorname{Ext}_{\mathcal{C}}^{1}(T, X)=0$ is equivalent to the fact that $X$ belongs to the additive subcategory add $T$ of $\mathcal{C}$. It is known that the combinatorics of cluster algebras are closely related to the combinatorics of cluster-tilting objects in triangulated 2-CalabiYau categories [8].

Definition 2.1. A realisable quadruple is a quadruple $(\mathcal{Q}, F, \mathcal{C}, T)$ such that

(a) $(\mathcal{Q}, F)$ is an ice quiver;

(b) $\mathcal{C}$ is a Hom-finite triangulated 2-Calabi-Yau category whose cluster-tilting objects form a cluster structure in the sense of [8];

(c) $T$ is a cluster-tilting object in $\mathcal{C}$;

(d) the ordinary quiver of $B_{T}=\operatorname{End}_{\mathcal{C}}(T)$ is $\mathcal{Q}$.

Following Reiten [50], every algebra of the form $\operatorname{End}_{\mathcal{C}}(T)$ as above is called a 2-Calabi-Yau tilted algebra. In order to simplify terminology, a category $\mathcal{C}$ satisfying condition (b) is simply called a triangulated 2-Calabi-Yau category.

Definition 2.2. Given a realisable quadruple $(\mathcal{Q}, F, \mathcal{C}, T)$, a $B_{T}$-module $M$ is called

(a) unfrozen if $M(i) \neq 0$ implies $i \notin F$;

(b) unfrozen indecomposable if it is unfrozen and indecomposable as a $B_{T}$-module;

(c) unfrozen sincere if $M(i) \neq 0$ if and only if $i \notin F$.

2.2. Examples. The notion of realisable quadruple covers a lot of situations in the context of cluster algebras. We now list some examples of such situations.

EXAMPLE 2.3. Let $Q$ be an acyclic quiver and $\mathcal{C}$ be the cluster category of $Q$, first defined in [10] (see also [15] for an alternative description in Dynkin type A). It is equipped canonically with a structure of triangulated 2-Calabi-Yau category $[\mathbf{1 0}, 35]$ and for any cluster-tilting object $T$ in $\mathcal{C}$, an algebra of the form $\operatorname{End}_{\mathcal{C}}(T)$ is called a cluster-tilted algebra, as defined in [11] (see also [15]). Thus, if $Q_{T}$ is the ordinary quiver of the cluster-tilted algebra $\operatorname{End}_{\mathcal{C}}(T)$, the quadruple $\left(Q_{T}, \emptyset, \mathcal{C}, T\right)$ is realisable and every $B_{T}$-module is unfrozen.

EXAMPLE 2.4. Let $(Q, W)$ be a Jacobi-finite quiver with potential, that is a quiver with potential in the sense of [22] such that the Jacobian algebra $\mathcal{J}_{(Q, W)}$ is finite dimensional. Let $\mathcal{C}_{(Q, W)}$ be the generalised cluster category constructed by Amiot [1, Section 3]. Then $\mathcal{C}_{(Q, W)}$ is a triangulated 2-Calabi-Yau category and there exists a cluster-tilting object $T$ in $\mathcal{C}_{(Q, W)}$ such that $\left(Q, \emptyset, \mathcal{C}_{(Q, W)}, T\right)$ is a realisable quadruple $[\mathbf{1}$, Theorem 3.6].

This example is of particular interest for the study of cluster algebras arising from surfaces in the sense of [27] (see also Section 6.2 for more details). Indeed, Labardini [40] associated a non-degenerate Jacobi-finite quiver with potential $(Q, W)$ to any marked surface $(S, M)$ with non-empty boundary. Thus, the generalised cluster category $\mathcal{C}_{(Q, W)}$ provides a categorification for the cluster algebra $\mathcal{A}(S, M)$ associated with the surface. Moreover, if the surface is unpunctured, that is if there are no marked points in the interior of the surface, it is known that the Jacobian algebra $\mathcal{J}_{(Q, W)}$ is a string algebra 
(it is, in fact, a gentle algebra, see [2]). The $\mathcal{J}_{(Q, W)}$-modules without self-extension are thus string modules and it follows from [31] that the cluster variables in $\mathcal{A}(S, M)$ can be studied via cluster characters associated with string $\mathcal{J}_{(Q, W)}$-modules (see Section 3 for details).

EXAMPLE 2.5. We now give a fundamental example with a non-empty set of frozen points. For any quiver $Q$, we denote by $\left(Q^{\text {pp }}, Q_{0}^{\prime}\right)$ its principal extension. It is defined as follows. We fix a copy $Q_{0}^{\prime}=\left\{i^{\prime} \mid i \in Q_{0}\right\}$ of $Q_{0}$ and set $Q_{0}^{\mathrm{pp}}=Q_{0} \sqcup Q_{0}^{\prime}$. The arrows in $Q_{1}^{\mathrm{pp}}$ between two points in $Q_{0}$ are the same as in $Q$ and for any $i \in Q_{0}$ we add an extra arrow $i^{\prime} \longrightarrow i$ in $Q_{1}^{\mathrm{pp}}$. Thus, the matrix of $Q^{\mathrm{pp}}$ is given by

$$
B\left(Q^{\mathrm{pp}}\right)=\left[\begin{array}{cc}
B(Q) & -I_{Q_{0}^{\prime}} \\
I_{Q_{0}^{\prime}} & 0
\end{array}\right],
$$

where $I_{Q_{0}^{\prime}}$ denotes the identity matrix in $M_{Q_{0}^{\prime}}(\mathbb{Z})$.

Now, if $Q$ is the ordinary quiver of a cluster-tilted algebra $B_{T}$, it is known that there exists a 2-Calabi-Yau category $\mathcal{C}^{\text {pp }}$, endowed with a cluster-tilting object $T^{\mathrm{pp}}$, obtained via the process of principal gluing, such that $\left(Q^{\mathrm{pp}}, Q_{0}^{\prime}, \mathcal{C}^{\mathrm{pp}}, T^{\mathrm{pp}}\right)$ is a realisable quadruple for which every $B_{T}$ module can naturally be viewed as an unfrozen module (see [31, Section 6.3] or Corollary 2.11).

2.3. Blown-up ice quivers and their realisations. In the proof of the main theorem of this paper, we are interested in a particular family of ice quivers, called blown-up. We now give some details concerning these quivers.

Definition 2.6. We say that an ice quiver $(\mathcal{Q}, F)$ is blown-up if $\mid \mathcal{Q}_{1}(i,-) \cup$ $\mathcal{Q}_{1}(-, i) \mid \leq 1$ for every point $i \in F$, that is if there exists at most one arrow starting or ending at any frozen point.

EXAMPLE 2.7. (a) Any ice quiver with an empty set of frozen points is blown-up.

(b) If $Q$ is any quiver, then its principal extension $\left(Q^{\mathrm{pp}}, Q_{0}^{\prime}\right)$ defined in Example 2.5 is blown-up.

REMARK 2.8. Any blown-up ice quiver $(\mathcal{Q}, F)$ whose unfrozen part is acyclic can be embedded in a realisable quadruple $(\mathcal{Q}, F, \mathcal{C}, T)$. Indeed, as $(\mathcal{Q}, F)$ is blown-up with an acyclic unfrozen part, $\mathcal{Q}$ is also acyclic. Thus, let $\mathcal{C}$ be the cluster category of the quiver $\mathcal{Q}$. Then the path algebra $\mathbf{k} \mathcal{Q}$ is identified with a cluster-tilting object in $\mathcal{C}$ and the corresponding cluster-tilted algebra is isomorphic to $\mathbf{k} \mathcal{Q}$ so that its ordinary quiver is $\mathcal{Q}$. Thus $(\mathcal{Q}, F, \mathcal{C}, \mathbf{k} \mathcal{Q})$ is a realisable quadruple.

We can construct a wide class of examples of realisable quadruples with the following proposition, due to Amiot [1].

Proposition 2.9. If $\mathcal{C}_{1}$ and $\mathcal{C}_{2}$ are two generalised cluster categories associated with Jacobi-finite quivers with potentials $\left(Q_{1}, W_{1}\right)$ and $\left(Q_{2}, W_{2}\right)$, then for any matrix $C$ with non-negative integer entries, there exists a Jacobi-finite quiver with potential $\left(Q^{\prime}, W^{\prime}\right)$ whose generalised cluster category $\mathcal{C}_{\left(Q^{\prime}, W^{\prime}\right)}$ has a cluster-tilting object $T^{\prime}$ such that the matrix associated with the ordinary quiver of the 2-Calabi-Yau tilted algebra 
$\operatorname{End}_{\mathcal{C}_{\left(Q^{\prime}, W^{\prime}\right)}}\left(T^{\prime}\right)$ is

$$
B^{\prime}=\left[\begin{array}{cc}
B\left(Q_{1}\right) & -C^{t} \\
C & B\left(Q_{2}\right)
\end{array}\right]
$$

Corollary 2.10. Let $(\mathcal{Q}, F)$ be a blown-up ice quiver with unfrozen part $Q$. Assume that there exists a potential $W$ on $Q$ such that the Jacobian algebra $\mathcal{J}_{(Q, W)}$ is finite dimensional. Then there exists a triangulated 2-Calabi-Yau category $\mathcal{C}$ and a clustertilting object $T$ in $\mathcal{C}$ such that $(\mathcal{Q}, F, \mathcal{C}, T)$ is a realisable quadruple.

Proof. We let $\mathcal{C}^{(0)}$ be the generalised cluster category associated with the quiver with potential $(Q, W)$. According to Example 2.4, there exists a cluster-tilting object $T^{(0)}$ in $\mathcal{C}$ such that the quadruple $\left(Q, \emptyset, \mathcal{C}^{(0)}, T^{(0)}\right)$ is realisable.

In order to simplify notations, we write $F=\{1, \ldots, m\}$. For any $i \in F$, we denote by $\mathcal{C}_{\mathbb{A}_{i}}$ a copy of the cluster category of type $\mathbb{A}_{1}$, which is a particular case of generalised cluster category in the sense of Amiot [1]. We now construct, by induction on $i \in$ $\{1, \ldots, m\}$, a category $\mathcal{C}^{(i)}$ with a cluster-tilting object $T^{(i)}$ such that the ordinary quiver of the 2-Calabi-Yau tilted algebra $\operatorname{End}_{\mathcal{C}^{(i)}}\left(T^{(i)}\right)$ is the full subquiver $(\mathcal{Q}, F)$ formed by points in $Q$ and frozen points in $\{1, \ldots, i\}$.

As $(\mathcal{Q}, F)$ is blown-up, for every $i \in\{1, \ldots, m\}$, there exists a unique arrow $\alpha_{i} \in$ $\mathcal{Q}_{1}(i,-) \sqcup \mathcal{Q}_{1}(-, i)$. If $\alpha_{i} \in \mathcal{Q}_{1}(i,-)$, we construct the category $\mathcal{C}^{(i)}$ gluing $\mathcal{C}^{(i-1)}$ and $\mathcal{C}_{\mathbb{A}_{i}}$ as provided by Proposition 2.9 with $\mathcal{C}_{1}=\mathcal{C}^{(i-1)}$ and $\mathcal{C}_{2}=\mathcal{C}_{A_{i}}$ and denote by $T^{(i)}$ the canonical cluster-tilting object in this gluing. If $\alpha_{i} \in \mathcal{Q}(-, i)$, we construct a gluing $\mathcal{C}^{(i)}$ of $\mathcal{C}_{\mathrm{A}_{i}}$ and $\mathcal{C}^{(i-1)}$ as provided by Proposition 2.9 with $\mathcal{C}_{1}=\mathcal{C}_{\mathrm{A}_{i}}$ and $\mathcal{C}_{2}=\mathcal{C}^{(i-1)}$ and denote by $T^{(i)}$ the canonical cluster-tilting object in this gluing. We finally set $\widetilde{\mathcal{C}}=\mathcal{C}^{(m)}$ and $\widetilde{T}=T^{(m)}$.

COROLlary 2.11. Let $(\mathcal{Q}, F)$ be a blown-up ice quiver whose unfrozen part is the ordinary quiver of a cluster-tilted algebra. Then $(\mathcal{Q}, F)$ can be embedded in a realisable quadruple $(\mathcal{Q}, F, \mathcal{C}, T)$.

Proof. It is proved in [36] and [9] that any cluster-tilted algebra is the Jacobian algebra of a Jacobi-finite quiver with potential. The result thus follows from Corollary 2.10 .

3. Cluster characters with coefficients. In this section we collect some background concerning Fu and Keller's cluster characters [31]. These characters allow one to give an explicit realisation of the elements in a cluster algebra in terms of the geometry and the homology underlying a 2-Calabi-Yau category.

We fix a triangulated 2-Calabi-Yau category $\mathcal{C}$ with suspension functor [1] and fix a cluster-tilting object $T$ in $\mathcal{C}$.

We let $\mathcal{Q}$ be the ordinary quiver of the 2-Calabi-Yau tilted algebra $B_{T}=\operatorname{End}_{\mathcal{C}}(T)$. Indecomposable direct summands of $T$ label the points in $\mathcal{Q}_{0}$. Let $F$ be a set of frozen points in $\mathcal{Q}_{0}$. We set $T_{F}=\bigoplus_{i \in F} T_{i}$. Consider the full subcategory $\mathcal{U}$ of $\mathcal{C}$ formed by the objects $X$ such that $\operatorname{Hom}_{\mathcal{C}}\left(T_{F}, X\right)=0$. Let (add $T$ [1]) be the ideal consisting of those morphisms factoring through objects of add $T[1]$.

TheOREM $3.1[\mathbf{1 1}, \mathbf{3 7}]$. The functor $\operatorname{Hom}_{\mathcal{C}}(T,-)$ induces an equivalence

$$
\operatorname{Hom}_{\mathcal{C}}(T,-): \mathcal{C} /(\operatorname{add} T[1]) \stackrel{\sim}{\rightarrow} \bmod -B_{T}
$$


The equivalence $\operatorname{Hom}_{\mathcal{C}}(T,-)$ induces an equivalence between $\mathcal{U} /(\operatorname{add} T[1])$ and the subcategory of mod- $B_{T}$ consisting of $B_{T}$-modules supported on the unfrozen part of $(\mathcal{Q}, F)$, which is denoted by $Q$. Slightly abusing notation, an object $M$ in $\mathcal{U} /(\operatorname{add} T[1])$ is identified with its image $\operatorname{Hom}_{\mathcal{C}}(T, M)$ in mod- $B_{T}$. Conversely, any $B_{T}$-module supported on $Q$ is viewed as an object in $\mathcal{U} /(\operatorname{add} T[1])$.

For any $i \in Q_{0}$, we denote by $S_{i}$ the simple $B_{T}$-module corresponding to the point $i$. We denote by $\langle-,-\rangle$ the truncated Euler form on mod- $B_{T}$ defined by

$$
\langle M, N\rangle=\operatorname{dim} \operatorname{Hom}_{B_{T}}(M, N)-\operatorname{dim} \operatorname{Ext}_{B_{T}}^{1}(M, N)
$$

for any $B_{T}$-modules $M$ and $N$. We denote by $\langle-,-\rangle_{a}$ the anti-symmetrised Euler form on $\bmod -B_{T}$ defined by

$$
\langle M, N\rangle_{a}=\langle M, N\rangle-\langle N, M\rangle
$$

for any $B_{T}$-modules $M$ and $N$.

Lemma 3.2 [46]. For any $i \in \mathcal{Q}_{0}$, the form $M \mapsto\left\langle S_{i}, M\right\rangle$ on mod- $B_{T}$ only depends on the class $[M]$ of $M$ in the Grothendieck group $K_{0}\left(\bmod -B_{T}\right)$ of $\bmod -B_{T}$.

For any $B_{T}$-module $M$ and any $\mathbf{e} \in K_{0}\left(\bmod -B_{T}\right)$, we let $\operatorname{Gr}_{\mathbf{e}}(M)$ denote the set of submodules $N$ of $M$ whose class $[N]$ in $K_{0}\left(\bmod -B_{T}\right)$ equals e. This set is called the Grassmannian of submodules of $M$ of dimension $\mathbf{e}$. It is a projective variety and we denote by $\chi\left(\operatorname{Gr}_{\mathbf{e}}(M)\right)$ its Euler-Poincaré characteristic (with respect to the singular cohomology if $\mathbf{k}$ is the field of complex numbers, and to the étale cohomology with compact support if $\mathbf{k}$ is arbitrary).

Definition 3.3 [46]. The cluster character associated with $(\mathcal{C}, T)$ is the unique map

$$
X_{?}^{T}: \mathrm{Ob}(\mathcal{C}) \longrightarrow \mathcal{L}\left(\mathbf{x}_{\mathcal{Q}}\right)
$$

such that

(a) $X_{T_{i}[1]}=x_{i}$ for any $i \in \mathcal{Q}_{0}$;

(b) if $M$ is indecomposable and not isomorphic to any $T_{i}[1]$,

$$
X_{M}^{T}=\sum_{\mathbf{e} \in \mathbb{N} \mathcal{Q}_{0}} \chi\left(\operatorname{Gr}_{\mathbf{e}}\left(\operatorname{Hom}_{\mathcal{C}}(T, M)\right)\right) \prod_{i \in \mathcal{Q}_{0}} x_{i}^{\left\langle S_{i}, \mathbf{e}\right\rangle_{a}-\left\langle S_{i}, \operatorname{Hom}_{\mathcal{C}}(T, M)\right\rangle} ;
$$

(c) for any two objects $M$ and $N$ in $\mathcal{C}$,

$$
X_{M \oplus N}^{T}=X_{M}^{T} X_{N}^{T}
$$

We recall that an indecomposable object $X$ in $\mathcal{C}$ is called reachable from $T$ if it is a direct summand of a cluster-tilting object that can be obtained from $T$ by a finite number of mutations (see [8]). In particular, any reachable object is rigid.

THEOREM 3.4 [31]. The map $X_{?}^{T}$ induces a surjection from the set of indecomposable objects in $\mathcal{C}$ which are reachable from $T$ to the set of cluster variables in the cluster algebra $\mathcal{A}(\mathcal{Q}, F)$. 
Note that identifying $\mathcal{C} /\left(\right.$ add $T$ [1]) with $\bmod -B_{T}$ using the functor $\operatorname{Hom}_{\mathcal{C}}(T,-)$, we associate to any indecomposable $B_{T}$-module $M$ the cluster character of an indecomposable lifting $\bar{M}$ of $M$ in $\mathcal{C} /($ add $T[1])$. Thus, we set

$$
X_{M}^{T}=X_{M}^{T}=\sum_{\mathbf{e} \in \mathbb{N} \mathcal{O}_{0}} \chi\left(\operatorname{Gr}_{\mathbf{e}}(M)\right) \prod_{i \in \mathcal{Q}_{0}} x_{i}^{\left\langle S_{i}, \mathbf{e}\right\rangle_{a}-\left\langle S_{i}, M\right\rangle},
$$

and this way, we may view $X_{?}^{T}$ as a map on the set of objects in $\bmod -B_{T}$.

4. A formula for Dynkin type $\mathbb{A}$ with coefficients. We now start the proof of our main result, which will be stated in Theorem 5.11. This section is devoted to the first step of the proof in which we establish the theorem for specific modules in the particular case of blown-up quivers with the unfrozen part of Dynkin type $\mathbb{A}$. The following is the result that we prove in this section.

THEOREM 4.1. Let $(\mathcal{Q}, F)$ be a blown-up ice quiver with unfrozen part $Q$ of Dynkin type $\mathbb{A}$ and $(\mathcal{Q}, F, \mathcal{C}, T)$ be a realisable quadruple. Let $B_{T}=\operatorname{End}_{\mathcal{C}}(T)$. Then for any indecomposable $B_{T}$-module $M$, the following hold:

(a) If $M$ is unfrozen, then it is a string module;

(b) If $M$ is a submodule of the unique unfrozen sincere module, then the corresponding cluster character is given by

$$
X_{M}^{T}=L_{M}
$$

Every $B_{T}$-module $M$ supported on $Q$ has the structure of a module over the path algebra $H=\mathbf{k} Q$ of the quiver $Q$. As $Q$ is of Dynkin type A, every such module $M$ is a string module if it is indecomposable. This proves the first point of the theorem.

In order to prove the second point, we need to collect the necessary background concerning the matrix product formula from [4], which we do in Section 4.1. Section 4.2 is devoted to the proof when $(\mathcal{Q}, F)$ is the principal extension of a Dynkin quiver of type A. In Section 4.3, we use the Fomin-Zelevinsky separation formula [28] in order to deduce the general case.

4.1. Background on the matrix product formula. Let $Q$ be a Dynkin quiver of type $\mathbb{A}_{n}$ with $n \geq 1$. Attach to each vertex $i$ of $Q_{0}$ a cluster variable $x_{i}$. As $Q$ is of Dynkin type $\mathbb{A}_{n}$, it is well known that the corresponding coefficient-free cluster algebra $\mathcal{A}(Q, \varnothing)$ is generated by $\frac{n(n+1)}{2}+n$ cluster variables. If $\mathcal{C}_{Q}$ denotes the cluster category of $Q$, then the set of indecomposable objects in $\mathcal{C}_{Q}$ can be identified with the disjoint union of the set of indecomposable $\mathbf{k} Q$-modules and $\left\{P_{i}[1] \mid i \in Q_{0}\right\}$, where $P_{i}$ denotes the indecomposable $\mathbf{k} Q$-module associated with point $i \in Q_{0}$. Identifying the path algebra $\mathbf{k} Q$ with a cluster-tilting object in $\mathcal{C}_{Q}$, the quadruple $\left(Q, \varnothing, \mathcal{C}_{Q}, \mathbf{k} Q\right)$ is realisable and the associated cluster character $M \mapsto X_{M}=X_{M}^{\mathbf{k} Q}$ induces a bijection from the set of indecomposable objects in $\mathcal{C}_{Q}$ to the set of cluster variables in $\mathcal{A}(Q, \varnothing)$. Moreover, this bijection sends each object of the form $P_{i}[1]$ onto $x_{i}$, and each indecomposable indecomposable $\mathbf{k} Q$-module $M$ onto the unique cluster variable having, in its reduced form, $\mathbf{x}^{\operatorname{dim} M}$ as a denominator, where $\operatorname{dim} M=\left(\operatorname{dim}_{\mathbf{k}} M(i)\right) \in \mathbb{N} Q_{0}$ (see [16]).

Under this identification, one can position the cluster variables of $\mathcal{A}(Q, \emptyset)$ into a grid underlying the Auslander-Reiten quiver of $\mathcal{C}_{Q}$. This positioning of variables on 
the grid corresponds to the frieze on the repetition quiver $\mathbb{Z} Q^{\text {op }}$ associated with the opposite quiver of $Q$ as constructed in [4]. We illustrate this by an example.

Let $Q$ be a Dynkin quiver of type $\mathbb{A}_{n}$, say

$$
1 \stackrel{\alpha}{\longrightarrow} 2 \stackrel{\beta}{\longrightarrow} 3 \stackrel{\gamma}{\longleftarrow} 4 \stackrel{\delta}{\longrightarrow} 5 \stackrel{\epsilon}{\longleftarrow} 6 \stackrel{\zeta}{\longleftarrow} 7 \stackrel{\eta}{\longleftarrow} 8 \stackrel{\theta}{\longrightarrow} 9 \stackrel{\iota}{\longrightarrow} 10 \stackrel{\kappa}{\longleftarrow} 11,
$$

(here $n=11$ ). The corresponding grid, in which we illustrated the cluster variables associated with the indecomposable projective $\mathbf{k} Q$-module $P_{i}$, and those associated with the indecomposable injective $\mathbf{k} Q$-module $I_{i}$, is as follows (the variable $X_{M(u, v)}$ will be explained thereafter):

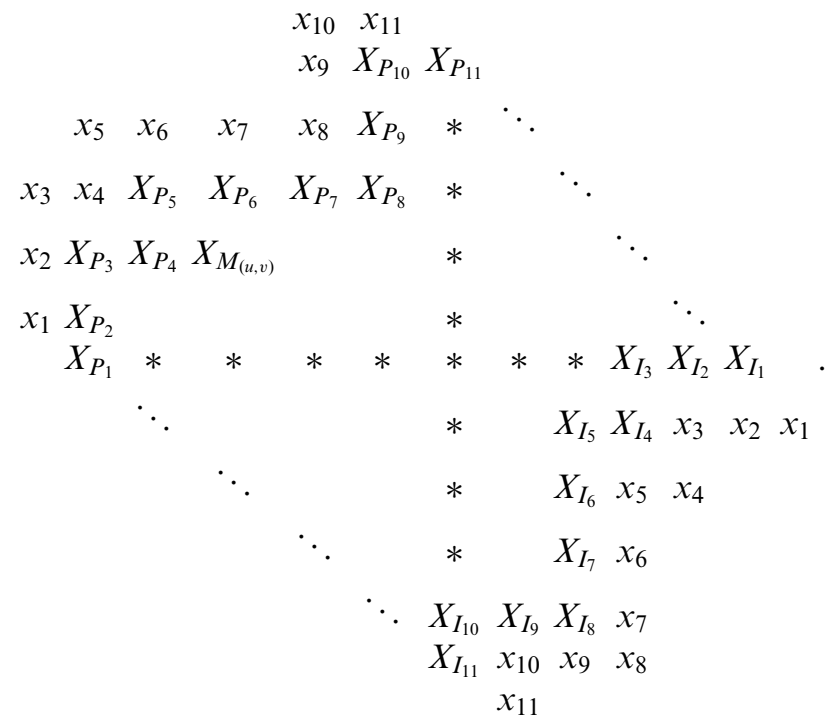

In [4, Section 8.2], the authors defined, for each cell $(u, v)$ in the grid, a Laurent polynomial $t_{Q^{\text {op }}}(u, v)$ whose definition depends on the region of the grid in which the cell is located.

For the purpose of our paper, it suffices to consider only the north-west component of the grid together with its south-east frontier of asterisks. It is important to observe that in mod-k $Q$, this part of the grid contains exactly all the indecomposable submodules of the unique indecomposable sincere $\mathbf{k} Q$-module, which is exactly positioned at the intersection of the vertical and the horizontal lines of asterisks (see for instance [33]). This observation will be crucial in Lemma 4.2 and Section 5.

We give more details on this region. For any cell $(u, v)$ located in the north-west component of the grid (and not on its south-east frontier of asterisks), its horizontal and vertical projections onto the initial variables $x_{i}$ determine a word $x_{k} x_{k+1} \cdots x_{k+l+1}$. Following [4], for each $j=1,2, \ldots, l-1$, let

$$
M\left(x_{j}, x_{j+1}\right)= \begin{cases}{\left[\begin{array}{cc}
x_{j} & 1 \\
0 & x_{j+1}
\end{array}\right]} & \text { if } x_{j} \text { is to the left of } x_{j+1}, \\
{\left[\begin{array}{cc}
x_{j+1} & 0 \\
1 & x_{j}
\end{array}\right]} & \text { if } x_{j} \text { is below } x_{j+1} .\end{cases}
$$


Then

$$
t_{Q^{\mathrm{op}}}(u, v)=\frac{1}{x_{k+1} \cdots x_{k+n}}\left[1, x_{k}\right]\left(\prod_{j=1}^{l-1} M\left(x_{k+j}, x_{k+j+1}\right)\right)\left[\begin{array}{c}
1 \\
x_{k+l+1}
\end{array}\right] .
$$

In order to determine the corresponding indecomposable $\mathbf{k} Q$-module $M_{(u, v)}$ for which $t_{Q^{\text {op }}}(u, v)=X_{M(u, v)}$, the horizontal and vertical projections onto the cluster variables $X_{P_{i}}$ gives the word $X_{P_{k+1}} X_{P_{k+2}} \cdots X_{P_{k+l}}$, meaning that $M_{(u, v)}$ is the string module corresponding to the unique string from the vertex $k+1$ to the vertex $k+l$ in $Q$. In our example, the cell $(u, v)$ gives rise to the word $x_{2} x_{3} \cdots x_{7}$, leading to

$$
t_{Q^{\mathrm{op}}}(u, v)=\frac{1}{x_{3} x_{4} x_{5} x_{6}}\left[1, x_{2}\right]\left[\begin{array}{cc}
x_{3} & 1 \\
0 & x_{4}
\end{array}\right]\left[\begin{array}{cc}
x_{5} & 0 \\
1 & x_{4}
\end{array}\right]\left[\begin{array}{cc}
x_{5} & 1 \\
0 & x_{6}
\end{array}\right]\left[\begin{array}{c}
1 \\
x_{7}
\end{array}\right],
$$

and $M_{(u, v)}$ corresponds to the string module whose corresponding string is $\gamma^{-1} \delta \epsilon^{-1}$ in $Q$.

Now a close inspection of the formulae (in the coefficient-free situation) gives $t_{Q^{\text {op }}}(u, v)=L_{M_{(u, v)}}$ whenever $(u, v)$ lies in the north-west region.

As mentioned above, we also need to consider the south-east frontier of asterisks of the north-west region. Observe that on this frontier $L_{M_{(u, v)}}$ is defined, while $t_{Q^{\text {op }}}(u, v)$ is not. In order to fix this, one can augment $Q^{\text {op }}$ with two new sinks, labeled 0 and $n+1$, in order to obtain a Dynkin quiver $\overline{Q^{\mathrm{op}}}$ of type $\mathbb{A}_{n+2}$ in such a way that the cells that were on the frontier of asterisks now lie in the north-west region in the grid corresponding to $\overline{Q^{\mathrm{op}}}$; thus $t_{Q^{\mathrm{op}}}(u, v)$ can be defined. In our example, it suffices to let $\overline{Q^{\mathrm{op}}}$ be given by

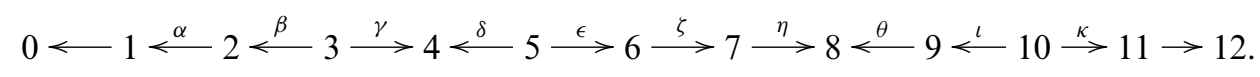

It is then easily checked that $L_{M_{(u, v)}}=\left.t_{\overline{Q^{\text {op }}}}(u, v)\right|_{\substack{x_{0}=1 \\ x_{n+1}=1}}$, that is $L_{M_{(u, v)}}$ is obtained from $t_{\overline{Q^{\mathrm{op}}}}(u, v)$ by specialising the initial cluster variables $x_{0}$ and $x_{n+1}$ to 1 . Observe that this relation also holds true for any cell located in the north-west region. So, in general, one can write

$$
L_{M_{(u, v)}}=\left.t \overline{Q^{\mathrm{op}}}(u, v)\right|_{\substack{x_{0}=1 \\ x_{n+1}=1}}
$$

whenever $(u, v)$ lies in the north-west region or on its south-east frontier of asterisks.

In the situation where we deal with arbitrary coefficients, let $(\mathcal{Q}, F)$ be an ice quiver with unfrozen part $Q$ of Dynkin type $\mathbb{A}$. Then generalising the coefficient-free situation, we let

$$
t_{Q^{\mathrm{op}}}(u, v)=L_{M_{(u, v)}} \text {. }
$$

For each $i \in Q_{0}$, let

$$
y_{i}=\prod_{\alpha \in \mathcal{Q}_{1}(F, i)} x_{s(\alpha)} \quad \text { and } \quad z_{i}=\prod_{\alpha \in \mathcal{Q}_{1}(i, F)} x_{t(\alpha)} .
$$

and for any $\mathbf{d}=\left(d_{i}\right)_{i \in Q_{0}} \in \mathbb{N} Q_{0}$, we set $\mathbf{y}^{\mathbf{d}}=\prod_{i \in Q_{0}} y_{i}^{d_{i}}$ and $\mathbf{z}^{\mathbf{d}}=\prod_{i \in Q_{0}} z_{i}^{d_{i}}$. 
Then a tedious and combinatorial adaptation of Lemmata 5 and 6 and Theorem 4 in [4], in which one needs to embed $Q^{\text {op }}$ in $\overline{Q^{\mathrm{op}}}$ as above, allows us to obtain the following recurrence relations, whose verification is left to the reader.

LEMMA 4.2.

(a) For any $i \in Q_{0}$, we have

$$
x_{i} L_{P_{i}}-y_{i}\left(\prod_{\alpha \in Q_{1}(i,-)} L_{P_{t(\alpha)}}\right)\left(\prod_{\alpha \in Q_{1}(-, i)} x_{s(\alpha)}\right)=\mathbf{z}^{\operatorname{dim} P_{i}} .
$$

(b) For any non-projective indecomposable submodule $M$ of the unique indecomposable unfrozen sincere $\mathbf{k} Q$-module, we have

$$
L_{\tau M} L_{M}-L_{E}=\mathbf{y}^{\operatorname{dim} \tau M} \mathbf{z}^{\operatorname{dim} M},
$$

where $E$ is the middle term of the almost split exact sequence $0 \longrightarrow \tau M \stackrel{i}{\rightarrow} E \stackrel{p}{\rightarrow}$ $M \longrightarrow 0$ in $\bmod -\mathbf{k} Q$.

4.2. Proof of Theorem 4.1. First step: principal coefficients. We start with two lemmata concerning cluster characters associated with principal extensions of acyclic quivers. These are analogues to [14, Lemma 3.9 and Proposition 3.10] (see also [26, Lemma 2.2]), which provide representation-theoretical interpretations of certain exchange relations in a cluster algebra with principal coefficients at an acyclic seed.

Let $Q$ be an acyclic quiver and $H=\mathbf{k} Q$ be the path algebra of $Q$, which is finite dimensional and hereditary. The map sending an $H$-module to its dimension vector allows us to identify the Grothendieck group $K_{0}(\bmod -H)$ with $\mathbb{Z}^{Q_{0}}$. If $(\mathcal{Q}, F)$ is the principal extension ( $Q^{\mathrm{pp}}, Q_{0}^{\prime}$ ) of $Q$ defined in Example 2.5, we denote by $X_{?}^{\mathrm{pp}}$ the corresponding cluster character and by $L^{\mathrm{pp}}$ the matrix formula of equation (1.1). For every $i^{\prime} \in Q_{0}^{\prime}$, we set $y_{i}=x_{i^{\prime}}$. It is known that

$$
X_{M}^{\mathrm{pp}}=\sum_{\mathbf{e} \in \mathbb{N} Q_{0}} \chi\left(\operatorname{Gr}_{\mathbf{e}}(M)\right) \prod_{i \in Q_{0}} x_{i}^{-\left\langle\mathbf{e},\left[S_{i}\right]\right\rangle-\left\langle\left[S_{i}\right],[M]-\mathbf{e}\right\rangle} y_{i}^{m_{i}-e_{i}},
$$

where the Euler forms and the Grassmannian are considered in mod- $H$ and $[M]=$ $\left(m_{i}\right)_{i \in Q_{0}}$ (see for instance [26, Remark 2.4]).

Lemma 4.3. Let $Q$ be an acyclic quiver. Then for any $i \in Q_{0}$, we have

$$
x_{i} X_{P_{i}}^{\mathrm{pp}}-y_{i}\left(\prod_{\alpha \in Q_{1}(i,-)} X_{P_{t(\alpha)}}^{\mathrm{pp}}\right)\left(\prod_{\alpha \in Q_{1}(-, i)} x_{s(\alpha)}\right)=1,
$$

where $P_{j}$ is the indecomposable projective $\mathbf{k} Q$-module associated with the vertex $j \in Q_{0}$.

Proof. The proof is a straightforward adaptation of the proof given in $[\mathbf{1 4}$, Lemma 3.9]; here we give it for completeness. We recall that for any $i \in Q_{0}$, we have

$$
\operatorname{Rad} P_{i}=\bigoplus_{\alpha \in Q_{1}(i,-)} P_{t(\alpha)} \text { and thus } X_{\operatorname{Rad} P_{i}}^{\mathrm{pp}}=\prod_{\alpha \in Q_{1}(i,-)} X_{P_{t(\alpha)}}^{\mathrm{pp}} .
$$


We also recall that $P_{i} / \operatorname{Rad} P_{i} \simeq S_{i}$ and that a submodule $M$ of $P_{i}$ either equals $P_{i}$ or is a submodule of $\operatorname{Rad} P_{i}$. We set $\operatorname{dim} P_{i}=\mathbf{m}=\left(m_{j}\right)_{j \in Q_{0}}$ and let $\delta$ be such that $\delta_{i j}=1$ if $i=j$ and $\delta_{i j}=0$ otherwise. Thus,

$$
\begin{aligned}
X_{\operatorname{Rad} P_{i}}^{\mathrm{pp}} & =\sum_{\mathbf{e}} \chi\left(\operatorname{Gr}_{\mathbf{e}}\left(\operatorname{Rad} P_{i}\right)\right) \prod_{l \in Q_{0}} x_{l}^{-\left\langle\mathbf{e},\left[S_{l}\right]\right\rangle-\left\langle\left[S_{l}\right], \mathbf{m}-\left[S_{i}\right]-\mathbf{e}\right\rangle} y_{l}^{m_{l}-\delta_{i l}-e_{l}} \\
& =\sum_{\mathbf{e}} \chi\left(\operatorname{Gr}_{\mathbf{e}}\left(\operatorname{Rad} P_{i}\right)\right) \prod_{l \in Q_{0}}\left(x_{l}^{-\left\langle\mathbf{e},\left[S_{l}\right]\right\rangle-\left\langle\left[S_{l}\right], \mathbf{m}-\mathbf{e}\right\rangle} y_{l}^{m_{l}-e_{l}}\right) x_{l}^{\left\langle\left[S_{l}\right],\left[S_{i}\right]\right\rangle} y_{l}^{-\delta_{i l}} \\
& =y_{i}^{-1}\left(\prod_{\alpha \in Q_{1}(-, i)} x_{s(\alpha)}^{-1}\right) x_{i} \sum_{\mathbf{e}} \chi\left(\operatorname{Gr}_{\mathbf{e}}\left(\operatorname{Rad} P_{i}\right)\right) \prod_{l \in Q_{0}} x_{l}^{-\left\langle\mathbf{e},\left[S_{l}\right]\right\rangle-\left\langle\left[S_{l}\right], \mathbf{m}-\mathbf{e}\right\rangle} y_{l}^{m_{l}-e_{l}},
\end{aligned}
$$

but

$$
X_{P_{i}}^{\mathrm{pp}}=x_{i}^{-1}+\sum_{\mathbf{e}} \chi\left(\mathrm{Gr}_{\mathbf{e}}\left(\operatorname{Rad} P_{i}\right)\right) \prod_{l \in Q_{0}} x_{l}^{-\left\langle\mathbf{e},\left[S_{l}\right]\right\rangle-\left\langle\left[S_{l}\right], \mathbf{m}-\mathbf{e}\right\rangle} y_{l}^{m_{l}-e_{l}} .
$$

Thus,

$$
x_{i} X_{P_{i}}^{\mathrm{pp}}=y_{i}\left(\prod_{\alpha \in Q_{1}(-, i)} x_{s(\alpha)}\right) X_{\operatorname{Rad} P_{i}}^{\mathrm{pp}}+1
$$

from which we deduce (4.4).

Lemma 4.4. Let $Q$ be an acyclic quiver. Then for any non-projective $\mathbf{k} Q$-module $M$, we have

$$
X_{\tau M}^{\mathrm{pp}} X_{M}^{\mathrm{pp}}-X_{E}^{\mathrm{pp}}=\mathbf{y}^{\operatorname{dim} \tau M},
$$

where $E$ is the central term of the almost split exact sequence $0 \longrightarrow \tau M \stackrel{i}{\rightarrow} E \stackrel{p}{\rightarrow} M \longrightarrow 0$.

Proof. This result is an analogue of [14, Proposition 3.10]. We sketch the proof for the convenience of the reader.

We write $N=\tau M, \operatorname{dim} N=\mathbf{n}=\left(n_{l}\right)_{l \in Q_{0}}$ and $\operatorname{dim} M=\mathbf{m}=\left(m_{l}\right)_{l \in Q_{0}}$. It follows from the definition of the character that we have

$$
X_{N}^{\mathrm{pp}} X_{M}^{\mathrm{pp}}=X_{N \oplus M}^{\mathrm{pp}}=\sum_{\mathbf{e} \in \mathbb{N} \varrho_{0}} \chi\left(\operatorname{Gr}_{\mathbf{e}}(N \oplus M)\right) \prod_{l \in Q_{0}} x_{l}^{-\left\langle\mathbf{e},\left[S_{l}\right]\right\rangle-\left\langle\left[S_{l}\right], \mathbf{n}+\mathbf{m}-\mathbf{e}\right\rangle} y_{l}^{m_{l}+n_{l}-e_{l}},
$$

but there is a surjective map with affine fibres

$$
\operatorname{Gr}_{\mathbf{e}}(N \oplus M) \longrightarrow \bigsqcup_{\mathbf{f}+\mathbf{g}=\mathbf{e}} \operatorname{Gr}_{\mathbf{f}}(N) \times \operatorname{Gr}_{\mathbf{g}}(M)
$$

so that $\chi\left(\operatorname{Gr}_{\mathbf{e}}(N \oplus M)\right)=\sum_{\mathbf{f}+\mathbf{g}=\mathbf{e}} \chi\left(\operatorname{Gr}_{\mathbf{f}}(N)\right) \chi\left(\operatorname{Gr}_{\mathbf{g}}(M)\right)$; thus

$$
X_{N \oplus M}^{\mathrm{pp}}=\sum_{\mathbf{f}, \mathbf{g}} \chi\left(\operatorname{Gr}_{\mathbf{f}}(N)\right) \chi\left(\operatorname{Gr}_{\mathbf{g}}(M)\right) \prod_{l \in Q_{0}} x_{l}^{-\left\langle\mathbf{f}+\mathbf{g},\left[S_{l}\right]\right\rangle-\left\langle\left[S_{l}\right], \mathbf{n}+\mathbf{m}-\mathbf{f}-\mathbf{g}\right\rangle} y_{l}^{m_{l}+n_{l}-f_{l}-g_{l}} .
$$


Now it follows from [14, Lemma 3.11] that every fibre of the map

$$
\zeta:\left\{\begin{aligned}
\operatorname{Gr}_{\mathbf{e}}(N \oplus M) & \longrightarrow \bigsqcup \bigsqcup_{\mathbf{f}+\mathbf{g}=\mathbf{e}} \operatorname{Gr}_{\mathbf{f}}(N) \times \operatorname{Gr}_{\mathbf{g}}(M) \\
U & \mapsto\left(i^{-1}(U), p(U)\right)
\end{aligned}\right.
$$

is an affine space except over the point $(0, M)$, where it is empty. It thus follows that

$$
X_{N \oplus M}^{\mathrm{pp}}=X_{E}^{\mathrm{pp}}+\prod_{l \in Q_{0}} y_{l}^{n_{l}},
$$

which establishes (4.5).

We now prove the second point of Theorem 4.1 for principal coefficients.

Proposition 4.5. Let $Q$ be a Dynkin quiver of type $\mathbb{A}$. Then for any indecomposable submodule $M$ of the unique unfrozen sincere $\mathbf{k} Q$-module, we have

$$
X_{M}^{\mathrm{pp}}=L_{M}^{\mathrm{pp}}
$$

Proof. The proof directly follows from Lemmata 4.2, 4.3 and 4.4, keeping in mind that for principal coefficients we have $z_{i}=1$ for each $i$ in Lemma 4.2.

4.3. Proof of Theorem 4.1 - second step. We now finish the proof of Theorem 4.1. For this, the strategy is to apply the Fomin-Zelevinsky [28] separation formula to the equality established in Proposition 4.5. We let $(\mathcal{Q}, F)$ be a blown-up ice quiver with unfrozen part $Q$ of Dynkin type $\mathbb{A}$ and we fix a realisable quadruple $(\mathcal{Q}, F, \mathcal{C}, T)$ and an unfrozen indecomposable $B_{T}$-module $M$, which has a natural structure of $H$-module, where $H$ is the path algebra of $Q$. We denote the principal extension of $Q$ by $\left(Q^{\text {pp }}, Q_{0}^{\prime}\right)$. Every $H$-module can naturally be viewed as a $B_{T}$-module or as a $\mathbf{k} Q^{\mathrm{pp}}$-module. According to Proposition 4.5, we know that $L_{M}^{\mathrm{pp}}=X_{M}^{\mathrm{pp}}$. We now want to prove that $L_{M}=X_{M}^{T}$, where $L_{\text {? }}$ denotes the matrix formula associated with $(\mathcal{Q}, F)$ in equation (1.1).

In order to simplify the notations, we identify $Q_{0}$ with $\{1, \ldots, n\}$ and for every $i \in Q_{0}$, we denote $i^{\prime}$ by $n+i$. Let $\mathbb{P}$ be the tropical semifield generated by the $x_{i}$, with $i \in F$, and endowed with the auxiliary addition

$$
\prod_{i} x_{i}^{a_{i}} \oplus \prod_{i} x_{i}^{b_{i}}=\prod_{i} x_{i}^{\min \left\{a_{i}, b_{i}\right\}} .
$$

For any $i \in Q_{0}$, we set

$$
w_{i}=\left(\prod_{\alpha \in \mathcal{Q}_{1}(F, i)} x_{s(\alpha)}\right)\left(\prod_{\alpha \in \mathcal{Q}_{1}(i, F)} x_{t(\alpha)}^{-1}\right) .
$$

With the notations of Section 4.1, we can thus write $w_{i}=y_{i} z_{i}^{-1}$. Following [29], for every subtraction-free rational expression $f$ in variables $x_{1}, \ldots, x_{2 n}$, we define the separation of $f$ as

$$
\sigma(f)=\frac{f\left(x_{1}, \ldots, x_{n}, w_{1}, \ldots, w_{n}\right)}{\left.f\right|_{\mathbb{P}}\left(1, \ldots, 1, w_{1}, \ldots, w_{n}\right)},
$$


where $\left.f\right|_{\mathbb{P}}$ means that we have replaced the ordinary addition in $\mathcal{F}\left(\mathbf{x}_{F}\right)$ by the auxiliary addition $\oplus$ of the semifield $\mathbb{P}$. This can be done because $f$ is subtraction-free.

Now we note that for every $H$-module, $L_{M}^{\mathrm{pp}}$ is a subtraction-free rational expression by definition. Moreover, for every indecomposable $H$-module $M, X_{M}^{\mathrm{pp}}$ is a cluster variable so that it is defined with a finite number of mutations, which are all subtractionfree. Thus, $X_{M}^{\mathrm{pp}}$ is also a subtraction-free rational expression (see [29, Section 3]) and we can apply $\sigma$ to both $X_{M}^{\mathrm{pp}}$ and $L_{M}^{\mathrm{pp}}$. As $L_{M}^{\mathrm{pp}}=X_{M}^{\mathrm{pp}}$ by Proposition 4.5 , we have $\sigma\left(L_{M}^{\mathrm{pp}}\right)=\sigma\left(X_{M}^{\mathrm{pp}}\right)$. Thus, we only need to prove that $\sigma\left(X_{M}^{\mathrm{pp}}\right)=X_{M}^{T}$ and $\sigma\left(L_{M}^{\mathrm{pp}}\right)=L_{M}$.

The equality $\sigma\left(X_{M}^{\mathrm{pp}}\right)=X_{M}^{T}$ follows directly from [29, Theorem 3.7] because $X_{\text {? }}^{\mathrm{pp}}$ and $X_{?}^{T}$ induce bijections from the set of indecomposable $H$-modules to the set of cluster variables in the cluster algebras $\mathcal{A}\left(Q^{\mathrm{pp}}, Q_{0}^{\prime}\right)$ and $\mathcal{A}(\mathcal{Q}, F)$, respectively, and these bijections respect denominator vectors.

The equality $\sigma\left(L_{M}^{\mathrm{pp}}\right)=L_{M}$ is obtained from the following observation. We write $c=\mathbf{s}(M)$. For each $j \in F, x_{j}$ appears in exactly one $w_{i}$, as there is exactly one $i \in Q_{0}$, which is adjacent to $j$ in $\mathcal{Q}$. On the other hand, in the product of matrices $L_{M}^{\mathrm{pp}}, x_{n+i}$ appears in at most one matrix, namely in the matrix $V_{c}^{\mathrm{pp}}(i)$ (where $V_{c}^{\mathrm{pp}}(i)$ denotes the matrices arising in the matrix product $\left.L_{c}^{\mathrm{pp}}\right)$. Thus, using the definition of the addition in $\mathbb{P}$ and the fact that $L_{M}^{\mathrm{pp}}\left(1, \ldots, 1, x_{n+1}, \ldots, x_{2 n}\right)$ has constant term 1 , it follows that

$$
\left.L_{M}^{\mathrm{pp}}\right|_{\mathbb{P}}\left(1, \ldots, 1, w_{1}, \ldots, w_{n}\right)=\prod_{i \in(\operatorname{supp}(M))_{0}} \prod_{\alpha \in Q_{1}^{\mathrm{pp}}(i, F)} x_{t(\alpha)}^{-1}
$$

Now, as

$$
\left(\prod_{\alpha \in \mathcal{Q}_{1}(i, F)} x_{t(\alpha)}\right)\left(V_{c}^{\mathrm{pp}}(i)\left(x_{1}, \ldots, x_{n}, w_{1}, \ldots, w_{n}\right)\right)=V_{c}(i),
$$

we get $\sigma\left(L_{M}^{\mathrm{pp}}\right)=L_{M}$. This ends the proof of Theorem 4.1.

5. The general case. We now deduce the main theorem from Theorem 4.1. For this, we use some 'blow-up' techniques that are described in Section 5.1. These techniques introduce some 'error' in the computation of the characters but this can be controlled with a normalising factor that we introduce in Section 5.2. The main result (Theorem 5.11) is stated in Section 5.4.

5.1. Blowing up quivers along string modules. In this section, we fix a quiver $Q$, denote by $M$ a string representation of $Q$ and write $c=\mathbf{s}(M)$. If $c$ is of positive length $n \geq 1$, we write $c=c_{1} \cdots c_{n}$.

The blow-up $\widetilde{Q_{M}}$ of $Q$ along $M$ is the quiver constructed as follows. Let $\left\{v_{1}, \ldots, v_{n+1}\right\}$ be a set. For any $i \in\{1, \ldots, n\}$, we set $\beta_{i}$ from $v_{i}$ to $v_{i+1}$, which is an arrow (or a formal inverse of an arrow, respectively) in $\left(\widetilde{Q_{M}}\right)_{1}$ if $c_{i}$ is an arrow (or a formal inverse of an arrow, respectively) in $Q_{1}$. For any $i \in\{1, \ldots, n\}$ and for any arrow $\alpha \in Q_{1}$ such that $\alpha \neq c_{i}^{ \pm 1}, c_{i-1}^{ \pm 1}$, if $s(\alpha)=t\left(c_{i}\right)$ (or $t(\alpha)=s\left(c_{i}\right)$, respectively), we create a new point, denoted by $t(\alpha)^{\alpha ; i}$ (or $s(\alpha)^{\alpha ; i}$, respectively) and an arrow $\alpha_{v_{i}}: v_{i} \longrightarrow t(\alpha)^{\alpha ; i}$ (or $\alpha_{v_{i}}: s(\alpha)^{\alpha ; i} \longrightarrow v_{i}$, respectively). 
EXAMPLE 5.1. Consider the quiver

$$
Q: \quad 1 \stackrel{\alpha}{\longrightarrow} 2 \underset{\gamma}{\stackrel{\epsilon}{\longrightarrow}} 3 \stackrel{\delta}{\longrightarrow} 4
$$

and let $M$ be the string representation corresponding to the walk $c=\epsilon^{-1} \gamma$, that is

$$
M: \quad 0 \longrightarrow \mathbf{k} \frac{[1,0]^{t}}{[0,1]^{t}} \mathbf{k}^{2} \longrightarrow 0 .
$$

Then, the quiver $\widetilde{Q_{M}}$ is

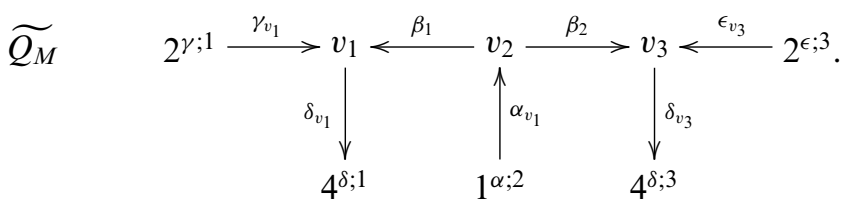

We recall the following definition from [34].

Definition 5.2. Let $Q$ and $S$ be two quivers. A winding of quivers $\Phi: Q \longrightarrow S$ is a pair $\Phi=\left(\Phi_{0}, \Phi_{1}\right)$, where $\Phi_{0}: Q_{0} \longrightarrow S_{0}$ and $\Phi_{1}: Q_{1} \longrightarrow S_{1}$ such that

(a) $\Phi$ is a morphism of quivers, that is $s \circ \Phi_{1}=\Phi_{0} \circ s$ and $t \circ \Phi_{1}=\Phi_{0} \circ t$.

(b) If $\alpha, \alpha^{\prime} \in Q_{1}$ with $\alpha \neq \alpha^{\prime}$ and $s(\alpha)=s\left(\alpha^{\prime}\right)$, then $\Phi_{1}(\alpha) \neq \Phi_{1}\left(\alpha^{\prime}\right)$.

(c) If $\alpha, \alpha^{\prime} \in Q_{1}$ with $\alpha \neq \alpha^{\prime}$ and $t(\alpha)=t\left(\alpha^{\prime}\right)$, then $\Phi_{1}(\alpha) \neq \Phi_{1}\left(\alpha^{\prime}\right)$.

With the above notations, the maps

$$
\Phi_{0}:\left\{\begin{aligned}
v_{i} & \mapsto t\left(c_{i-1}\right) & & \text { for any } i \in\{1, \ldots, n+1\} \\
v^{\alpha ; i} & \mapsto v & & \text { for any } v \in \overline{\operatorname{supp}(M)} \text { and any } \alpha, i
\end{aligned}\right.
$$

and

$$
\Phi_{1}:\left\{\begin{aligned}
\beta_{i} \mapsto c_{i} & \text { for any } i \in\{1, \ldots, n\} \\
\alpha_{v_{i}} \mapsto \alpha & \text { for any arrow of the form } \alpha_{v_{i}}
\end{aligned}\right.
$$

induce a winding of quivers $\Phi: \widetilde{Q_{M}} \longrightarrow \overline{\operatorname{supp}(M)}$.

Let $\Phi_{*}$ be the map from the set of objects in $\operatorname{rep}\left(\widetilde{Q_{M}}\right)$ to the set of objects in $\operatorname{rep}(\overline{\operatorname{supp}(M)})$ that associates to a representation $\widetilde{V}$ of $\widetilde{Q_{M}}$ the representation $V$ given by

$$
V(i)=\bigoplus_{j \in \Phi_{0}^{-1}(i)} \tilde{V}(j) \text { and } V(\alpha)=\bigoplus_{\beta \in \Phi_{1}^{-1}(\alpha)} \tilde{V}(\beta)
$$

for any $i \in(\overline{\operatorname{supp}(M)})_{0}$ and any $\alpha \in(\overline{\operatorname{supp}(M)})_{1}$. At the level of dimension vectors, $\Phi_{*}$ induces a natural map $\phi: \mathbb{N}^{\left(\widetilde{Q_{M}}\right)_{0}} \longrightarrow \mathbb{N}^{(\overline{\operatorname{supp}(M)})_{0}}$.

We define a representation $\widetilde{M}$ of $\widetilde{Q_{M}}$ by setting for any point $v \in\left(\widetilde{Q_{M}}\right)_{0}$

$$
\widetilde{M}(v)= \begin{cases}\mathbf{k} & \text { if } v=v_{i} \text { for some } i \in\{0, \ldots, n\} . \\ 0 & \text { otherwise }\end{cases}
$$


and for any arrow $\alpha \in\left(\widetilde{Q_{M}}\right)_{1}$

$$
\widetilde{M}(\alpha)= \begin{cases}1_{\mathbf{k}} & \text { if } \alpha=\beta_{i}^{ \pm 1} \text { for some } i \in\{1, \ldots, n\} \\ 0 & \text { otherwise. }\end{cases}
$$

Lemma 5.3. Let $B$ be a finite dimensional k-algebra. Then for any string B-module $M$, we have $\Phi_{*}(\tilde{M}) \simeq M$.

Proof. This follows from the construction.

Definition 5.4. If $B$ is a finite dimensional k-algebra with bound quiver $(Q, I)$ and $M$ is a $B$-module, the border $\partial M$ of $M$ is the set of points in the closure $\overline{\operatorname{supp}(M)}$ of the support of $M$ in $Q$, which do not lie in the support $\operatorname{supp}(M)$ of $M$.

Thus, with the above notations, the border $\partial \widetilde{M}$ consists of all the points in $\left(\widetilde{Q_{M}}\right)_{0}$, which do not lie in the support of $\widetilde{M}$.

LEMMA 5.5. Let B be a finite dimensional k-algebra. Then for any string B-module $M$, the pair $\left(\widetilde{Q_{M}}, \partial \widetilde{M}\right)$ is a blown-up ice quiver whose unfrozen part is of Dynkin type $\mathbb{A}$. Moreover, the representation $\widetilde{M}$ is a sincere unfrozen string representation of $\widetilde{Q_{M}}$.

Proof. The first assertion follows from the construction of $\widetilde{Q_{M}}$ and $\widetilde{M}$. For the second assertion, we observe that $\widetilde{M}$ is supported on the unfrozen part of $\widetilde{Q_{M}}$, which is of Dynkin type A. It is unfrozen sincere and indecomposable by construction so that it is a string representation.

EXAMPLE 5.6. Let $B$ be the path algebra of the quiver $Q$ and $M$ be the string module considered in Example 5.1. Then the representation $\widetilde{M}$ of the quiver $\widetilde{Q_{M}}$ is

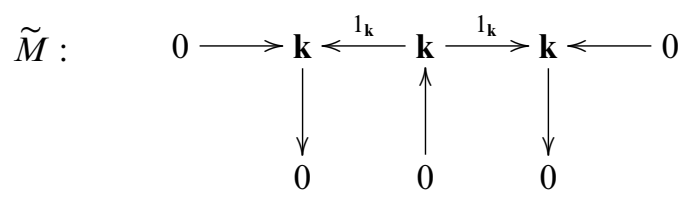

so that $\left(\widetilde{Q_{M}}, \partial \widetilde{M}\right)$ is indeed a blown-up ice quiver with unfrozen part of Dynkin type $A_{3}$.

5.2. Normalisation. In this section $M$ denotes a string module over a finite dimensional algebra $B$ with bound quiver $(Q, I)$.

Definition 5.7. The normalising vector of $M$ is $\mathbf{n}_{M}=\left(n_{i}\right)_{i \in \operatorname{supp}(M)_{0}} \in \mathbb{N} \overline{\operatorname{supp}(M)_{0}}$ given by

$$
n_{i}=\left\langle S_{i}, M\right\rangle-\sum_{j \in \Phi_{0}^{-1}(i)}\left\langle S_{j}, \tilde{M}\right\rangle
$$

for any $i \in \overline{\operatorname{supp}(M)_{0}}$, where the first truncated Euler form is considered in mod- $B$ and the second truncated Euler form is considered in mod-k $\widetilde{Q_{M}}$. 
The normalising factor of $M$ is

$$
\mathbf{x}^{\mathbf{n}_{M}}=\prod_{i \in \operatorname{supp}(M)_{0}} x_{i}^{n_{i}} .
$$

This normalising vector is actually easy to compute in several usual situations as it is explained in Section 7.

EXAMPLE 5.8. Consider the finite dimensional algebra $B$ whose ordinary quiver is

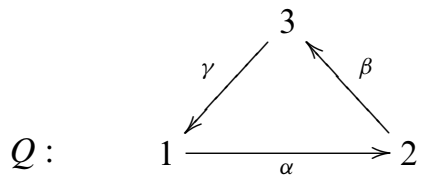

and whose relations are given by the vanishing of all paths of length two, that is $\alpha \beta=\beta \gamma=\gamma \alpha=0$. It is a cluster-tilted algebra of type $\mathbb{A}_{3}$. Consider the projective module $M$ associated with point 1 . It is a string $B$-module with string $\alpha$. The associated blown-up quiver $\widetilde{Q_{M}}$ is $3^{\gamma ; 1} \longrightarrow v_{1} \longrightarrow v_{2} \longrightarrow 3^{\beta ; 2}$ and the representation $\widetilde{M}$ is $0 \longrightarrow \mathbf{k} \stackrel{1_{\mathbf{k}}}{\rightarrow} \mathbf{k} \longrightarrow 0$. Then, one has $\left\langle S_{1}, M\right\rangle=\left\langle S_{v_{1}}, \tilde{M}\right\rangle=0,\left\langle S_{2}, M\right\rangle=\left\langle S_{v_{2}}, \tilde{M}\right\rangle=1$ and $\left\langle S_{3}, M\right\rangle=0$, whereas $\left\langle S_{3 \beta ; 2}, \widetilde{M}\right\rangle+\left\langle S_{3 \gamma ; 1}, \tilde{M}\right\rangle=0-1=-1$. Thus, the normalising vector is $\mathbf{n}_{M}=(0,0,1)$.

5.3. Blow-ups and cluster characters. We keep the notations of Section 5.1. As usual, we naturally identify $\mathcal{L}\left(\mathbf{x}_{\overline{\operatorname{supp}(M)}}\right)$ to a subring of $\mathcal{L}\left(\mathbf{x}_{\mathcal{Q}}\right)$. We consider the following surjective morphism of $\mathbb{Z}$-algebras:

$$
\pi: \mathcal{L}\left(\mathbf{x}_{\widetilde{Q_{M}}}\right) \longrightarrow \mathcal{L}\left(\mathbf{x}_{\overline{\operatorname{supp}(M)}}\right)
$$

defined by

$$
\pi\left(x_{v_{i}}\right)=x_{t\left(c_{i-1}\right)}
$$

for any $1 \leq i \leq n+1$ and

$$
\pi\left(x_{v^{\alpha ; i}}\right)=x_{v}
$$

for any $v \in \overline{\operatorname{supp}(M)}$ and any $\alpha, i$.

We now observe that for any unfrozen string module $M$ with respect to a realisable quadruple $(\mathcal{Q}, F, \mathcal{C}, T)$, the Laurent polynomial $X_{M}^{T}$ is in the image of the function $\pi$.

LEMMA 5.9. Let $(\mathcal{Q}, F, \mathcal{C}, T)$ be a realisable quadruple and $M$ be an unfrozen string module. Then

$$
X_{M}^{T} \in \mathcal{L}\left(\mathbf{x}_{\overline{\operatorname{supp}(M)}}\right)
$$

Proof. We recall that unfrozen $B_{T}$-modules are identified via $\operatorname{Hom}_{\mathcal{C}}(T,-)$ with the objects $M$ in $\mathcal{U} /($ add $T[1])$. We have

$$
X_{M}^{T}=\sum_{\mathbf{e} \in \mathbb{N}^{\mathcal{Q}_{0}}} \chi\left(\operatorname{Gr}_{\mathbf{e}}(M)\right) \prod_{i \in \mathcal{Q}_{0}} x_{i}^{\left\langle S_{i}, \mathbf{e}\right\rangle_{a}-\left\langle S_{i}, M\right\rangle} .
$$


Any dimension vector e such that $\operatorname{Gr}_{\mathbf{e}}(M) \neq \emptyset$ is supported on $\operatorname{supp}(M)$. Thus, if $i \in \mathcal{Q}_{0}$ is not in $\overline{\operatorname{supp}(M)}$, then $\left\langle S_{i}, \mathbf{e}\right\rangle_{a}=0$ and $\left\langle S_{i}, M\right\rangle=0$. In particular,

$$
X_{M}^{T}=\sum_{\mathbf{e} \in \mathbb{N}_{0}} \chi\left(\operatorname{Gr}_{\mathbf{e}}(M)\right) \prod_{i \in \overline{\operatorname{supp}(M)}} x_{i}^{\left\langle S_{i}, \mathbf{e}\right\rangle_{a}-\left\langle S_{i}, M\right\rangle} \in \mathcal{L}\left(\mathbf{x}_{\overline{\operatorname{supp}(M)}}\right)
$$

Let $\widetilde{\mathcal{C}}$ be the cluster category of the quiver $\widetilde{Q_{M}}$ and $\widetilde{T}=\mathbf{k} \widetilde{Q_{M}}$ be the path algebra of $\widetilde{Q_{M}}$, which is identified with a cluster-tilting object in $\widetilde{\mathcal{C}}$. We denote by $\widetilde{X}_{\text {? }}$ the corresponding cluster character with values in $\mathcal{L}\left(\mathbf{x}_{\widetilde{Q}_{M}}\right)$.

Proposition 5.10. Let $(\mathcal{Q}, F, \mathcal{C}, T)$ be a realisable quadruple and $M$ be an unfrozen string module. Then,

$$
\pi\left(\widetilde{X}_{\widetilde{M}}\right)=\mathbf{x}^{\mathbf{n}_{M}} X_{M}^{T}
$$

Proof. We have

$$
\begin{aligned}
X_{M}^{T} & =\sum_{\mathbf{e} \in \mathbb{N}^{\mathcal{Q}_{0}}} \chi\left(\operatorname{Gr}_{\mathbf{e}}(M)\right) \prod_{i \in \mathcal{Q}_{0}} x_{i}^{\left\langle S_{i}, \mathbf{e}\right\rangle_{a}-\left\langle S_{i}, M\right\rangle} \\
& =\sum_{\mathbf{e} \in \mathbb{N}^{\operatorname{supp}(M)_{0}}} \chi\left(\operatorname{Gr}_{\mathbf{e}}(M)\right) \prod_{i \in(\overline{\operatorname{supp}(M)})_{0}} x_{i}^{\left\langle S_{i}, \mathbf{e}\right\rangle_{a}-\left\langle S_{i}, M\right\rangle} .
\end{aligned}
$$

Now, for any e $\in \mathbb{N}^{\operatorname{supp}(M)_{0}}$, as $\Phi$ is a winding of quivers and $\Phi_{*}(\tilde{M})=M$, it follows from [34, Theorem $1.2(\mathrm{a})]$ that

$$
\chi\left(\operatorname{Gr}_{\mathbf{e}}(M)\right)=\sum_{\mathbf{f} \in \phi^{-1}(\mathbf{e})} \chi\left(\operatorname{Gr}_{\mathbf{f}}(\tilde{M})\right)
$$

Fix $\mathbf{e} \in \mathbb{N}^{\operatorname{supp}(M)_{0}}$ and $\mathbf{f} \in \phi^{-1}(\mathbf{e})$. We now prove that for any $i \in \overline{\operatorname{supp}(M)}$, we have

$$
x_{i}^{\left\langle S_{i}, \mathbf{e}\right\rangle_{a}}=\pi\left(\prod_{j \in \Phi_{0}^{-1}(i)} x_{j}^{\left\langle S_{j}, \mathbf{f}\right\rangle_{a}}\right)
$$

where the anti-symmetrised Euler form on the left-hand side is taken in mod-k $\widetilde{Q_{M}}$ and the anti-symmetrised Euler forms on the right-hand side are taken in mod- $B$.

First note that

$$
\pi\left(\prod_{j \in \Phi_{0}^{-1}(i)} x_{j}^{\left\langle S_{j}, \mathbf{f}\right\rangle_{a}}\right)=x_{i}^{\sum_{j \in \Phi_{0}^{-1}(i)}\left\langle S_{j}, \mathbf{f}\right\rangle_{a}}
$$

so that it is enough to prove that

$$
\left\langle S_{i}, \mathbf{e}\right\rangle_{a}=\sum_{j \in \Phi_{0}^{-1}(i)}\left\langle S_{j}, \mathbf{f}\right\rangle_{a}
$$


For any module $U$ and any integer $n \geq 1$, we denote by $n U$ the direct sum of $n$ copies of $U$. Now, as $\left\langle S_{i},-\right\rangle_{a}$ is well defined on the Grothendieck group $K_{0}\left(\bmod -B_{T}\right)$ (Lemma 3.2), we have

$$
\begin{aligned}
\left\langle S_{i}, \mathbf{e}\right\rangle_{a} & =\sum_{k \in \operatorname{supp}(M)_{0}}\left\langle S_{i}, e_{k} S_{k}\right\rangle_{a} \\
& =\sum_{k \in \operatorname{supp}(M)_{0}}\left\langle S_{i},\left(\sum_{l \in \Phi_{0}^{-1}(k)} f_{l}\right) S_{k}\right\rangle_{a} \\
& =\sum_{k \in \operatorname{supp}(M)_{0}}\left(\sum_{l \in \Phi_{0}^{-1}(k)} f_{l}\right)\left\langle S_{i}, S_{k}\right\rangle_{a},
\end{aligned}
$$

and

$$
\begin{aligned}
\sum_{j \in \Phi_{0}^{-1}(i)}\left\langle S_{j}, \mathbf{f}\right\rangle_{a} & =\sum_{k \in \operatorname{supp}(M)_{0}} \sum_{l \in \Phi_{0}^{-1}(k)} \sum_{j \in \Phi_{0}^{-1}(i)} f_{l}\left\langle S_{j}, S_{l}\right\rangle_{a} \\
& =\sum_{k \in \operatorname{supp}(M)_{0}} \sum_{l \in \Phi_{0}^{-1}(k)} f_{l}\left(\sum_{j \in \Phi_{0}^{-1}(i)}\left\langle S_{j}, S_{l}\right\rangle_{a}\right) .
\end{aligned}
$$

But for any $k \in \operatorname{supp}(M)_{0}$ and any $l \in \Phi_{0}^{-1}(k)$, we have

$$
\sum_{j \in \Phi_{0}^{-1}(i)}\left\langle S_{j}, S_{l}\right\rangle_{a}=\left\langle S_{i}, S_{k}\right\rangle_{a}
$$

which proves (5.6).

Now,

$$
\widetilde{X}_{\widetilde{M}}=\sum_{\mathbf{f} \in \mathbb{N}\left(\widetilde{\left.Q_{M}\right)_{0}}\right.} \chi\left(\operatorname{Gr}_{\mathbf{f}}(\widetilde{M})\right) \prod_{i \in\left(\widetilde{Q_{M}}\right)_{0}} x_{i}^{\left\langle S_{i}, \mathbf{f}_{a}-\left\langle S_{i}, \widetilde{M}\right\rangle\right.},
$$

so that

$$
\begin{aligned}
& \pi\left(\widetilde{X}_{\widetilde{M}}\right)=\sum_{\mathbf{e} \in \mathbb{N} \overline{\mathbb{N u p p}^{\prime}\left(M_{0}\right)}} \sum_{\mathbf{f} \in \phi^{-1}(\mathbf{e})} \chi\left(\operatorname{Gr}_{\mathbf{f}}(\tilde{M})\right) \prod_{i \in \overline{\operatorname{supp}(M)_{0}}} \pi\left(\prod_{j \in \Phi_{0}^{-1}(i)} x_{j}^{\left\langle S_{j}, \mathbf{f}\right\rangle_{a}-\left\langle S_{j}, \widetilde{M}\right\rangle}\right) \\
& =\sum_{\mathbf{e} \in \mathbb{N}^{\operatorname{supp}(M)_{0}}}\left(\sum_{\mathbf{f} \in \phi^{-1}(\mathbf{e})} \chi\left(\operatorname{Gr}_{\mathbf{f}}(\tilde{M})\right)\right) \prod_{i \in \operatorname{supp}(M)_{0}} x_{i}^{\left\langle S_{i}, \mathbf{e}_{a}\right.} \pi\left(\prod_{i \in \Phi_{0}^{-1}(i)} x_{j}^{-\left\langle S_{j}, \widetilde{M}\right\rangle}\right) \\
& =\sum_{\mathbf{e} \in \mathbb{N}^{\operatorname{supp}(M)_{0}}} \chi\left(\operatorname{Gr}_{\mathbf{e}}(M)\right) \prod_{i \in \overline{\operatorname{supp}(M)_{0}}} x_{i}^{\left\langle S_{i},\right\rangle_{a}-\sum_{j \in \oplus_{0}^{-1}}\left\langle S_{j}, \widetilde{M}\right\rangle} \\
& =\mathbf{x}^{\mathbf{n}_{M}} X_{M}^{T} \text {. }
\end{aligned}
$$

This finishes the proof. 
5.4. The main theorem. We can now prove the main theorem of this paper.

THEOREM 5.11. Let $(\mathcal{Q}, F, \mathcal{C}, T)$ be a realisable quadruple and $M$ be an unfrozen string module with respect to this quadruple. Then

$$
X_{M}^{T}=\frac{1}{\mathbf{x}^{\mathbf{n}_{M}}} L_{M} .
$$

Proof. We first notice that with the above notations, it follows directly from the definitions that

$$
\pi\left(\widetilde{L}_{\widetilde{M}}\right)=L_{M}
$$

Thus,

$$
X_{M}^{T}=\frac{1}{\mathbf{x}^{\mathbf{n}_{M}}} \pi\left(\widetilde{X}_{\widetilde{M}}\right)=\frac{1}{\mathbf{x}^{\mathbf{n}_{M}}} \pi\left(\widetilde{L}_{\widetilde{M}}\right)=\frac{1}{\mathbf{x}^{\mathbf{n}_{M}}} L_{M},
$$

where the first equality follows from Proposition 5.10, the second one from Theorem 4.1 and Lemma 5.5 and the third from the above observation.

6. Applications. In this section we give several applications of Theorem 5.11.

6.1. Computing cluster variables. We now prove that the formula $M \mapsto L_{M}$ given in equation (1.1) allows one to compute the cluster variables in several situations.

Corollary 6.1. Let $(\mathcal{Q}, F)$ be an ice quiver. Assume that there exists a realisable quadruple $(\mathcal{Q}, F, \mathcal{C}, T)$ such that every rigid object in $\mathcal{C}$ is reachable from $T$. Then for any unfrozen $\operatorname{End}_{\mathcal{C}}(T)$-module $M$, which is rigid and indecomposable, the Laurent polynomial $\frac{1}{\mathbf{x}^{\mathbf{n}_{M}}} L_{M}$ is a cluster variable in $\mathcal{A}(\mathcal{Q}, F)$.

Proof. We identify $M$ with an indecomposable lifting in $\mathcal{C}$ for the functor $\operatorname{Hom}_{\mathcal{C}}(T,-)$, which is also rigid in $\mathcal{C}$ by $[32,54]$. It follows from Theorem 3.4 that the map $M \mapsto X_{M}^{T}$ induces a surjection from the set of reachable rigid objects into the set of cluster variables in $\mathcal{A}(\mathcal{Q}, F)$. It is also rigid in $\mathcal{C}$; hence, by hypothesis, it is reachable and thus $X_{M}^{T}$ is a cluster variable in $\mathcal{A}(\mathcal{Q}, F)$.

COROLlary 6.2. Let $B_{T}$ be a cluster-tilted algebra. Then for any rigid string $B_{T^{-}}$ module $M$, the Laurent polynomial $\frac{1}{\mathbf{x}^{n_{M}}} L_{M}$ is a cluster variable in the coefficient-free cluster algebra associated with the ordinary quiver of $B_{T}$.

Proof. Let $\mathcal{C}$ be a cluster category, $T$ be a cluster-tilting object in $\mathcal{C}$ and $Q_{T}$ be the ordinary quiver of $B_{T}$. Then the quadruple $\left(Q_{T}, \emptyset, \mathcal{C}, T\right)$ is realisable (see Example 2.3). Moreover, it follows from [10] that every indecomposable rigid object in $\mathcal{C}$ is reachable from $T$. Thus, the result follows from Corollary 6.1 .

COROLlary 6.3. Let $B_{T}$ be a cluster-tilted algebra of Dynkin type $\mathbb{A}$ or affine type 夰. Then, the map

$$
M \mapsto \frac{1}{\mathbf{x}^{\mathbf{n}_{M}}} L_{M}
$$


induces a bijection from the set of indecomposable rigid $B_{T}$-modules to the set of cluster variables in the coefficient-free cluster algebra associated with the ordinary quiver of $B_{T}$ that do not belong to the initial cluster.

Proof. Cluster-tilted algebras of Dynkin type $\mathbb{A}$ or affine type $\widetilde{A}$ are string algebras [2]. Thus, each indecomposable rigid $B_{T}$-module is a string module. Now, if $\mathcal{C}$ is a cluster category and $T$ is a cluster-tilting object in $\mathcal{C}$ such that $B_{T}=\operatorname{End}_{\mathcal{C}}(T)$, it follows in particular from Theorem 5.11 that for every indecomposable rigid $B_{T}$-module $M$, we have $X_{M}^{T}=\frac{1}{\mathrm{x}^{\mathrm{x}_{M}}} L_{M}$. But, then it follows from [46] that $M \mapsto \frac{1}{\mathrm{x}_{M}} L_{M}$ is a bijection from the set of indecomposable rigid $B_{T}$-modules to the set of cluster variables in the coefficient-free cluster algebra $\mathcal{A}\left(Q_{T}, \emptyset\right)$ that do not belong to the initial cluster $\mathbf{x}_{Q_{T}}$, where $Q_{T}$ denotes the ordinary quiver of $B_{T}$.

6.2. Positivity in cluster algebras. We now prove positivity results in cluster algebras using formula (1.1). We first recall the basic notions concerning positivity in cluster algebras.

If $\mathbb{P}$ is an Abelian group, we denote its group ring by $\mathbb{Z} \mathbb{P}$. Let $n \geq 1$ be an integer and $\mathbf{x}=\left(x_{1}, \ldots, x_{n}\right)$ be a $n$-tuple of indeterminates. We denote by $\mathbb{Z}_{\geq 0} \mathbb{P}\left[\mathbf{x}^{ \pm 1}\right]$ the semiring of the subtraction-free Laurent polynomials in the variables in $\mathbf{x}$ with coefficients in $\mathbb{Z} \mathbb{P}$, that is, the set of elements of the form

$$
\frac{f\left(x_{1}, \ldots, x_{n}\right)}{x_{1}^{d_{1}} \ldots x_{n}^{d_{n}}},
$$

where $f$ is a polynomial in $n$ variables whose coefficients are non-negative linear combinations of elements of $\mathbb{P}$ and $d_{1}, \ldots, d_{n} \in \mathbb{Z}$. In particular, in the case where $\mathbb{P}=\{1\}$, the notation $\mathbb{Z}_{\geq 0}\left[\mathbf{x}^{ \pm 1}\right]$ denotes the semiring of Laurent polynomials in variables $x_{1}, \ldots, x_{n}$ with non-negative coefficients.

If $Q$ is a quiver without loops and 2-cycles, $\mathbb{P}$ is any semifield and $\mathbf{y}$ is a $Q_{0}$-tuple of elements of $\mathbb{P}$, it follows from the Laurent phenomenon that every cluster variable in the cluster algebra $\mathcal{A}\left(Q, \mathbf{x}_{Q}, \mathbf{y}\right)$ is an element of $\mathbb{Z} \mathbb{P}\left[\mathbf{c}^{ \pm 1}\right]$ when expressed in any cluster $\mathbf{c}$ of $\mathcal{A}\left(Q, \mathbf{x}_{Q}, \mathbf{y}\right)$ [28]. The Fomin and Zelevinsky positivity conjecture, stated in [28], asserts that every cluster variable is actually an element of $\mathbb{Z}_{\geq 0} \mathbb{P}\left[\mathbf{c}^{ \pm 1}\right]$. This conjecture was proved for rank two cluster algebras [23, 43, 53], cluster algebras arising from

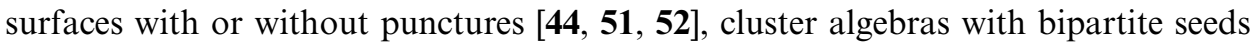
[45] and weaker versions were proved for acyclic cluster algebras $[4,45,49]$.

COROLlaRY 6.4. Let $(\mathcal{Q}, F, \mathcal{C}, T)$ be a realisable quadruple and $M$ be an unfrozen string module with respect to this quadruple. Then

$$
X_{M}^{T} \in \mathbb{Z}_{\geq 0}\left[\mathbf{x}_{\operatorname{supp}(M)}{ }^{ \pm 1}\right] .
$$

Proof. The set $\mathbb{Z}_{\geq 0}\left[\mathbf{x}_{\overline{\text { supp }(M)}}\right]$ is a semiring for the usual sum and product. Thus, the set $M_{2}\left(\mathbb{Z}_{\geq 0}\left[\mathbf{x}_{\overline{\operatorname{supp}(M)}}\right]\right)$ is also a semiring for the usual sum and product of matrices. It follows that $L_{M} \in \mathbb{Z}_{\geq 0}\left[\mathbf{x}_{\overline{\operatorname{supp}(M)}}{ }^{ \pm 1}\right]$ and by Theorem 5.11, $X_{M}^{T}=L_{M}$. This proves the corollary.

This corollary was also obtained by Cerulli and Haupt $[\mathbf{1 8}, \mathbf{3 4}]$. 
Corollary 6.5. Let $B_{T}$ be a cluster-tilted algebra. Then $X_{M}^{T} \in \mathbb{Z}_{\geq 0}\left[\mathbf{x}_{\overline{\operatorname{supp}(M)}}{ }^{ \pm 1}\right]$ for any string $B_{T}$-module $M$.

We now provide a new proof of the Fomin and Zelevinsky [28] positivity conjecture for cluster algebras arising from unpunctured surfaces. We first set some notations. If $(S, M)$ is an oriented, bordered and marked surface, Fomin, Shapiro and Thurston associated with any triangulation $\Gamma$ of $(S, M)$ a quiver $Q_{\Gamma}$ [27]. For any semifield $\mathbb{P}$ and any $\left(Q_{\Gamma}\right)_{0}$-tuple $\mathbf{y}$ of elements of $\mathbb{P}$, we denote by $\mathcal{A}\left(Q_{\Gamma}, \mathbf{x}_{Q_{\Gamma}}, \mathbf{y}\right)$ the cluster algebra with initial seed $\left(Q_{\Gamma}, \mathbf{x}_{Q_{\Gamma}}, \mathbf{y}\right)$ (see [29]). Such a cluster algebra does not depend on the choice of the triangulation $\Gamma$ of $(S, M)$, up to an isomorphism of $\mathbb{Z} \mathbb{P}$-algebras [27]. It is called the cluster algebra arising from the surface $(S, M)$ and is denoted by $\mathcal{A}(S, M)$.

For this class of cluster algebras, Fomin and Zelevinsky positivity conjecture amounts to saying that any cluster variable $z$ in $\mathcal{A}(S, M)$ belongs to $\mathbb{Z}_{\geq 0} \mathbb{P}\left[\mathbf{x}_{Q_{\Gamma}}^{ \pm 1}\right]$ for any choice of triangulation $\Gamma[\mathbf{2 8}$, Section 3]. This conjecture was proved in $[\mathbf{5 1}, \mathbf{5 2}]$ for unpunctured surfaces and in [44] for arbitrary surfaces. We now provide a new independent representation-theoretical proof of the positivity conjecture for cluster algebras arising from unpunctured surfaces.

COROLLARY 6.6. Let $(S, M)$ be an unpunctured surface. Then the positivity conjecture holds for $\mathcal{A}(S, M)$ equipped with an arbitrary choice of coefficients.

Proof. Using Fomin-Zelevinsky separation formula [29, Theorem 3.7], it is enough to prove the result for principal coefficients. Let $\Gamma$ be any triangulation of $(S, M)$ and $\left(Q_{\Gamma}, W_{\Gamma}\right)$ be the quiver with potential associated with this triangulation as in [2] (see also [40]). Let $\mathcal{J}_{\Gamma}=\mathcal{J}_{\left(Q_{\Gamma}, W_{\Gamma}\right)}$ be the corresponding Jacobian algebra, which is finite dimensional [2]. It follows from Corollary 2.10 that the principal extension $\left(Q_{\Gamma}^{\mathrm{pp}},\left(Q_{\Gamma}\right)_{0}^{\prime}\right)$ can be embedded in a realisable quadruple $\left(Q_{\Gamma}^{\mathrm{pp}},\left(Q_{\Gamma}\right)_{0}^{\prime}, \mathcal{C}, T\right)$. Now, let $z$ be a cluster variable in $\mathcal{A}(S, M)$, it follows from Theorem 3.4 that either $z$ belongs to the initial cluster $\mathbf{x}_{Q_{\Gamma}}$ or $z=X_{M}^{T}$ for some indecomposable rigid $\mathcal{J}_{\Gamma}$-module $M$. As the algebra $\mathcal{J}_{\Gamma}$ is a string algebra, any indecomposable rigid $\mathcal{J}_{\Gamma}$-module is a string module. Thus, if we set $y_{i}=x_{i^{\prime}}$ for any $i \in\left(Q_{T}\right)_{0}$, it follows from Corollary 6.4 that

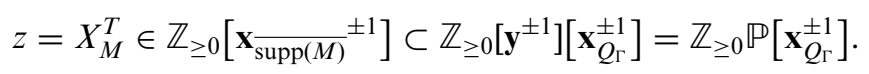

\section{3. $S L(2, \mathbb{Z})$ and total Grassmannians of submodules.}

Definition 6.7. Given a finite dimensional k-algebra $B$ and a finitely generated $B$-module $M$, the total Grassmannian of submodules of $M$ is the set

$$
\operatorname{Gr}(M)=\bigsqcup_{\mathbf{e} \in K_{0}(\bmod -B)} \operatorname{Gr}_{\mathbf{e}}(M) .
$$

As $M$ is finite dimensional, all but finitely many $\operatorname{Gr}_{\mathbf{e}}(M)$ are empty, so that $\operatorname{Gr}(M)$ is a finite disjoint union of projective varieties. It is thus endowed with a structure of projective variety and we can consider its Euler-Poincaré characteristic, $\chi(\operatorname{Gr}(M))=$ $\sum_{\mathbf{e}} \chi\left(\mathrm{Gr}_{\mathbf{e}}(M)\right) \in \mathbb{Z}$. 
For modules over hereditary algebras, these numbers can be computed with friezes as shown in [3, Section 4]. Here we prove that, for string modules over 2-Calabi-Yau tilted algebras, these numbers can be computed with products of matrices in $S L(2, \mathbb{Z})$.

If $Q$ is any quiver, for any $\beta \in Q_{1}$, we define two matrices in $\operatorname{SL}(2, \mathbb{Z})$ :

$$
a(\beta)=\left[\begin{array}{ll}
1 & 0 \\
1 & 1
\end{array}\right] \text { and } a\left(\beta^{-1}\right)=\left[\begin{array}{ll}
1 & 1 \\
0 & 1
\end{array}\right]
$$

and for any walk $c=c_{1} \cdots c_{n}$ in $Q$, we set

$$
l_{c}=\left[\begin{array}{ll}
1, & 1
\end{array}\right]\left(\prod_{i=1}^{n} a\left(c_{i}\right)\right)\left[\begin{array}{l}
1 \\
1
\end{array}\right] \in \mathbb{N} .
$$

Finally, if $c$ is a walk of length 0 , we set $l_{c}=2$.

PROPOSITION 6.8. For any string module $M$ over a finite dimensional algebra, we have

$$
\chi(\operatorname{Gr}(M))=l_{\mathbf{s}(M)} .
$$

Proof. Let $B$ be a finite dimensional algebra with ordinary quiver $Q$ and $M$ be a string $B$-module. We use the notations of Section 5.1 . Let $\widetilde{M}$ be the representation of the blow-up $\widetilde{Q_{M}}$ of $Q$ along $M$. Then $\widetilde{M}$ is a string representation of $\widetilde{Q_{M}}$ and we have $l_{\mathbf{s}(M)}=l_{\mathbf{s}(\widetilde{M})}$. Moreover, it follows from [34, Theorem 1.2 (a)] that

$$
\sum_{\mathbf{e} \in \mathbb{N} Q_{0}} \chi\left(\operatorname{Gr}_{\mathbf{e}}(M)\right)=\sum_{\mathbf{e} \in \mathbb{N} Q_{0}} \sum_{\mathbf{f} \in \phi^{-1}(\mathbf{e})} \chi\left(\operatorname{Gr}_{\mathbf{f}}(\tilde{M})\right)=\sum_{\mathbf{f} \in \mathbb{N}\left(\widetilde{Q_{M}}\right)_{0}} \chi\left(\operatorname{Gr}_{\mathbf{f}}(\tilde{M})\right),
$$

so that the characteristic of the total Grassmannian of $M$ equals the characteristic of the total Grassmannian of $\widetilde{M}$. As the Grassmannians of submodules of $\widetilde{M}$ only depend on the support of $\widetilde{M}$, it is enough to prove the proposition for indecomposable modules over path algebras of Dynkin quivers of type $\mathbb{A}$.

Thus, let $B$ be the path algebra of a Dynkin quiver $Q$ of type $\mathbb{A}, \mathcal{C}$ be the cluster category of $Q$ and $T=\mathbf{k} Q$. Then $(Q, \varnothing, \mathcal{C}, T)$ is a realisable quadruple such that $B=\operatorname{End}_{\mathcal{C}}(T)$. According to Theorem 5.11, we have

$$
X_{M}^{T}=\frac{1}{\mathbf{x}_{Q}^{\mathbf{n}_{M}}} L_{M}
$$

Consider the surjective morphism of $\mathbb{Z}$-algebras.

$$
p:\left\{\begin{aligned}
\mathcal{L}\left(\mathbf{x}_{Q}\right) & \longrightarrow \mathbb{Z} \\
x_{i} & \mapsto 1
\end{aligned} \quad \text { for any } i \in Q_{0} .\right.
$$

Then it follows from the definition of cluster character that $p\left(X_{M}^{T}\right)=\chi(\operatorname{Gr}(M))$.

We now prove that $p\left(L_{M}\right)=l_{\mathbf{s}(M)}$. If the string $\mathbf{s}(M)$ of $M$ is of zero length, then $M$ is simple and thus

$$
\chi(\operatorname{Gr}(M))=\chi\left(\operatorname{Gr}_{[0]}(M)\right)+\chi\left(\operatorname{Gr}_{[M]}(M)\right)=1+1=2=l_{\mathbf{s}(M)} .
$$


Otherwise, we write $\mathbf{s}(M)=c_{1} \cdots c_{n}$ with $n \geq 1$. Then, for any $i \in\{1, \ldots, n\}$, we have $p\left(A\left(c_{i}\right)\right)=a\left(c_{i}\right)$ and $p\left(V_{c}\left(s\left(c_{i}\right)\right)\right)=p\left(V_{c}\left(t\left(c_{i}\right)\right)\right)$ is the identity matrix. As $p$ is a ring homomorphism, it induces a ring homomorphism at the level of matrices and thus $p\left(L_{M}\right)=p\left(L_{c}\right)=l_{c}$. As $p\left(\frac{1}{\mathbf{x}_{Q}^{n_{M}}}\right)=1$, it follows that

$$
\chi(\operatorname{Gr}(M))=p\left(X_{M}^{T}\right)=p\left(L_{M}\right)=l_{\mathbf{s}(M)},
$$

and the proposition is proved.

EXAMPLE 6.9. Let $(S, M)$ be an unpunctured surface. Let $\Gamma$ be a triangulation of $(S, M),\left(Q_{\Gamma}, W_{\Gamma}\right)$ be the quiver with potential associated with this triangulation and $\mathcal{J}_{\Gamma}=\mathcal{J}_{\left(Q_{\Gamma}, W_{\Gamma}\right)}$ be the corresponding Jacobian algebra. Then for any string module $M$ over $\mathcal{J}_{\Gamma}$, we have $\chi(\operatorname{Gr}(M))=l_{\mathbf{s}(M)}$.

7. More about normalisation. We now describe some situations in which the normalising factor can be omitted or computed combinatorially.

7.1. The hereditary case. We first observe that the normalising factor can be omitted in several cases.

LEMMA 7.1. Let $(\mathcal{Q}, F, \mathcal{C}, T)$ be a realisable quadruple and $M$ be an unfrozen string module. If the full subcategory of $\bmod -B_{T}$ formed by all modules, which are supported on $\overline{\operatorname{supp}(M)}$, is hereditary, then $\mathbf{n}_{M}=0$.

Proof. Let $i \in{\overline{\operatorname{supp}(M)_{0}}}_{0}$, as $M$ and $S_{i}$ are supported on $\overline{\operatorname{supp}(\widetilde{M})}$, the truncated Euler form $\left\langle S_{i}, M\right\rangle$ only depends on dimension vectors. But, as $\widetilde{Q_{M}}$ is acyclic and $\sum_{j \in \Phi_{0}^{-1}(i)}\left\langle S_{j}, \widetilde{M}\right\rangle$ only depends on the dimension vectors, it follows that $\left\langle S_{i}, M\right\rangle=$ $\sum_{j \in \Phi_{0}^{-1}(i)}\left\langle S_{j}, \tilde{M}\right\rangle$ and thus $\mathbf{n}_{M}=0$.

COROLlary 7.2. Let $(\mathcal{Q}, F, \mathcal{C}, T)$ be a realisable quadruple such that $B_{T}$ is hereditary. Then $\mathbf{n}_{M}=0$ for every unfrozen string module $M$.

7.2. The maximal factor method for modules over cluster-tilted algebras. For any walk $c$ in a locally finite quiver $Q$ we set

$$
N_{c}=\left[\begin{array}{ll}
1, & 1
\end{array}\right]\left(\prod_{i=0}^{n} A\left(c_{i}\right) V_{c}(i+1)\right)\left[\begin{array}{l}
1 \\
1
\end{array}\right] \in \mathcal{L}\left(\mathbf{x}_{Q}\right),
$$

so that (1.1) becomes

$$
L_{c}=\frac{1}{\prod_{i=0}^{n} x_{t\left(c_{i}\right)}} N_{c}
$$

Let $\eta_{c} \in \mathbb{N}^{Q_{0}}$ such that

$$
N_{c}=\mathbf{x}_{Q}^{\eta_{c}} P_{c}\left(\mathbf{x}_{Q}\right)
$$

with $P_{c}\left(\mathbf{x}_{Q}\right)$ not divisible by any $x_{i}$ with $i \in Q_{0}$. 
Let $\mathcal{C}$ be a cluster category, $T=T_{1} \oplus \cdots \oplus T_{n}$ be a cluster-tilting object in $\mathcal{C}$, then $B_{T}=\operatorname{End}_{\mathcal{C}}(T)$ is a cluster-tilted algebra and we denote its ordinary quiver by $Q$. Then $(Q, \emptyset, \mathcal{C}, T)$ is a realisable quadruple and every $B_{T}$-module is unfrozen.

We now prove that for such modules, we can compute the normalising vector combinatorially.

Proposition 7.3. Consider the above notations and assume that $\operatorname{End}_{\mathcal{C}}\left(T_{i}\right) \simeq \mathbf{k}$ for any $i \in\{1, \ldots, n\}$. Then for every rigid string $B_{T}$-module $M$, we have $\mathbf{n}_{M}=$ $\eta_{\mathbf{s}(M)}$.

Proof. For any Laurent polynomial $L\left(x_{1}, \ldots, x_{n}\right) \in \mathbb{Z}\left[x_{1}^{ \pm 1}, \ldots, x_{n}^{ \pm 1}\right]$, we denote by $\delta(L) \in \mathbb{Z}^{n}$ its denominator vector, that is the unique vector $\left(d_{1}, \ldots, d_{n}\right)$ such that $L\left(x_{1}, \ldots, x_{n}\right)=f\left(x_{1}, \ldots, x_{n}\right) / \prod_{i=1}^{n} x_{i}^{d_{i}}$, where $f\left(x_{1}, \ldots, x_{n}\right)$ is a polynomial not divisible by any $x_{i}$.

$\operatorname{As~}_{\operatorname{End}}\left(T_{i}\right) \simeq \mathbf{k}$ for any $i \in\{1, \ldots, n\}$, it follows from [12] (see also [24]) that for any indecomposable rigid $B_{T}$-module

$$
\delta\left(X_{M}^{T}\right)=\left(\operatorname{dim} \operatorname{Hom}_{\mathcal{C}}\left(T_{i}, \bar{M}\right)\right)_{1 \leq i \leq n},
$$

where $\bar{M}$ is an indecomposable rigid object in $\mathcal{C}$ such that $\operatorname{Hom}_{\mathcal{C}}(T, \bar{M})=M$.

As $\operatorname{Hom}_{\mathcal{C}}(T,-)$ induces an equivalence of categories $\mathcal{C} /(\operatorname{add} T[1]) \simeq \bmod -B_{T}$, it follows that

$$
\delta\left(X_{M}^{T}\right)=\left(\operatorname{dim} \operatorname{Hom}_{B_{T}}\left(P_{i}, M\right)\right)_{1 \leq i \leq n},
$$

where $P_{i}$ is the indecomposable projective $B_{T}$-module at point $i$. For any $i \in\{1, \ldots, n\}$, the dimension of $\operatorname{Hom}_{B_{T}}\left(P_{i}, M\right)$ is the multiplicity of the simple $B_{T}$-module $S_{i}$ as a composition factor of $M$. If $M$ is a string module, this multiplicity is also equal to the number of occurrences of $i$ along the string $\mathbf{s}(M)$. Thus, if $\mathbf{s}(M)=c_{1} \cdots c_{n}$, the denominator of $X_{M}^{T}$ in its irreducible form is equal to $\prod_{i=0}^{n} x_{t\left(c_{i}\right)}$.

On the other hand, the denominator of $L_{M}$ (in its irreducible form) is $\mathbf{x}^{-\eta_{\mathbf{s}(M)}} \prod_{i=0}^{n} x_{t\left(c_{i}\right)}$. Now it follows from Theorem 5.11 that $X_{M}^{T}=\frac{1}{\mathbf{x}^{n_{M}}} L_{M}$ and thus the denominators coincide, that is to say $\mathbf{n}_{M}=\eta_{\mathbf{s}(M)}$.

\section{Examples.}

8.1. First example in type $\mathbb{A}_{2}$. We start with a very simple and detailed example, which is nevertheless instructive in order to understand the behaviour of the normalising vector.

We consider the frozen quiver $(\mathcal{Q}, F)$ where

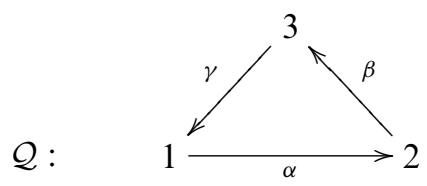

and $F=\{3\}$. 
The corresponding cluster algebra is of geometric type with initial seed $(\mathbf{x}, \mathbf{y}, B)$, where $\mathbf{x}=\left(x_{1}, x_{2}\right), \mathbf{y}=\left(x_{3}\right)$ and

$$
B=\left[\begin{array}{rr}
0 & 1 \\
-1 & 0 \\
1 & -1
\end{array}\right]
$$

Its unfrozen part $Q$ is of Dynkin type $\mathbb{A}_{2}$. Note that $\mathcal{Q}$ is the ordinary quiver of a cluster-tilted algebra of type $\mathbb{A}_{3}$ whose relations are $\alpha \beta=\beta \gamma=\gamma \alpha=0$. Thus, $(\mathcal{Q}, F)$ can be embedded in a realisable quadruple $(\mathcal{Q}, F, \mathcal{C}, T)$, where $\mathcal{C}$ is a cluster category of type $\mathbb{A}_{3}$. There are three indecomposable modules supported on $Q$, which are the simple $S_{1}$, the simple $S_{2}$ and the projective $\mathbf{k} Q$-module $P_{1}$, and all these modules are string modules.

As $(\mathcal{Q}, F)$ is not blown-up (there are two arrows entering or leaving the frozen point 3 ), we cannot simply apply Theorem 4.1 . So we apply Theorem 5.11 , which means that we need to consider normalising factors.

The string of $S_{1}$ is $e_{1}$ and therefore applying formula (1.1) to $S_{1}$ we get $L_{S_{1}}=\frac{x_{2}+x_{3}}{x_{1}}$. The quiver $\widetilde{Q_{S_{1}}}$ is $3^{\gamma ; 1} \longrightarrow v_{1} \longrightarrow 2^{\alpha ; 1}$ and thus it is easily computed that $\mathbf{n}_{S_{1}}=0$ so that $X_{S_{1}}=L_{S_{1}}$, which is indeed the cluster variable in $\mathcal{A}(\mathcal{Q}, F)$ corresponding to the simple module $S_{1}$. Similarly, we prove that $X_{S_{2}}=L_{S_{2}}=\frac{x_{1}+x_{3}}{x_{2}}$.

The case of $P_{1}$ is slightly more instructive. We apply the matrix formula and get $L_{P_{1}}=\frac{x_{3}\left(x_{1}+x_{2}+x_{3}\right)}{x_{1} x_{2}}$. We can now use Proposition 7.3 in order to conclude that the normalising factor is $x_{3}$, but we do the computation in order to see the blowup technique working. The quiver $\overline{\operatorname{supp}\left(P_{1}\right)}$ is $\mathcal{Q}$ itself. Its blow-up along $P_{1}$ is thus $\widetilde{Q_{P_{1}}}: 3^{\gamma ; 1} \longrightarrow v_{1} \longrightarrow v_{2} \longrightarrow 3^{\beta ; 2}$ and $\widetilde{M}$ is the representation $\widetilde{P}_{1}: 0 \longrightarrow \mathbf{k} \stackrel{1_{\mathbf{k}}}{\rightarrow} \mathbf{k} \longrightarrow 0$. We compute that

$$
\widetilde{L}_{\widetilde{P}_{1}}=\frac{x_{v_{1}} x_{3 \gamma ; 1}+x_{v_{2}} x_{3^{\beta ; 2}}+x_{3 \gamma ; 1} x_{3^{\beta ; 2}}}{x_{v_{1}} x_{v_{2}}},
$$

so that the morphism $\pi$ sending $x_{v_{1}}$ to $x_{1}, x_{v_{2}}$ to $x_{2}$ and $x_{3^{\gamma ; 1}}, x_{3^{\beta ; 2}}$ to $x_{3}$ satisfies $\pi\left(\widetilde{L}_{\widetilde{M}}\right)=$ $L_{M}$. Moreover, computing directly, or applying Theorem 4.1 , we get $\widetilde{X}_{\widetilde{M}}=\widetilde{L}_{\widetilde{P}_{1}}$.

The normalising vector of $M$ is $\mathbf{n}_{M}=(0,0,1)$ (see Example 5.8) so that we get $X_{P_{1}}=\frac{1}{x_{3}} L_{P_{1}}=\frac{x_{1}+x_{2}+x_{3}}{x_{1} x_{2}}$, which is indeed the cluster variable in $\mathcal{A}(\mathcal{Q}, F)$ corresponding to this module.

8.2. Regular cluster variables in type $\widetilde{\mathbb{A}}$. Let $Q$ be a Euclidean quiver of type $\widetilde{\mathbb{A}}$ equipped with an acyclic orientation. In [4, Theorem 4], the authors provided a combinatorial formula for expressing all but finitely many cluster variables in the coefficient-free cluster algebra associated with $Q$. The cluster variables they computed, in fact, correspond to the cluster characters associated with indecomposable postprojective $\mathbf{k} Q^{\mathrm{op}}$-modules. Dualising their methods, it is possible to compute the cluster characters associated with indecomposable preinjective $\mathbf{k} Q^{\mathrm{op}}$-modules. Their methods do not allow one to compute the remaining cluster variables, namely those corresponding to regular rigid $\mathbf{k} Q^{\mathrm{op}}$-modules. We now use Theorem 4.1 to complete the formula and compute cluster variables associated with regular modules.

It is known that the Auslander-Reiten quiver of mod-k $Q^{\text {op }}$ contains at most two exceptional tubes (that is tubes of rank $\geq 2$ ) and all the rigid indecomposable regular 
modules belong to these tubes. Every indecomposable regular module (rigid or not) in such a tube is a string module and its string may be described completely. For simplicity, we only compute the cluster variables associated with quasi-simple regular modules in such tubes, this can easily be extended to any module in one of the exceptional tubes. It is well known (see for instance [3, Section 5.2]) that any such module is of the form

$$
\cdots 0 \stackrel{0}{\longleftarrow} \mathbf{k} \stackrel{1_{k}}{\longrightarrow} \mathbf{k} \stackrel{1_{k}}{\longrightarrow} \cdots \stackrel{1_{k}}{\longrightarrow} \mathbf{k} \stackrel{1_{k}}{\longrightarrow} \mathbf{k} \stackrel{0}{\longleftarrow} 0 \cdots
$$

so that if we depict locally the quiver $Q^{\text {op }}$ by

$$
\cdots 0<1 \stackrel{\alpha_{1}}{\longrightarrow} 2 \stackrel{\alpha_{2}}{\longrightarrow} \cdots \stackrel{\alpha_{n-2}}{\longrightarrow} n-1 \stackrel{\alpha_{n-1}}{\longrightarrow} n<n+1 \cdots,
$$

the string is $c=\alpha_{1} \cdots \alpha_{n-1}$ and we compute

$$
L_{c}=\frac{1}{\prod_{i=1}^{n+1} x_{i}}\left[\begin{array}{ll}
1, & 1
\end{array}\right]\left[\begin{array}{cc}
x_{0} & 0 \\
0 & 1
\end{array}\right]\left[\begin{array}{cc}
\prod_{i=1}^{n-1} x_{i+1} & 0 \\
\sum_{j=1}^{n-1} \frac{\prod_{i=1}^{n} x_{i}}{x_{j} x_{j+1}} & \prod_{i=1}^{n-1} x_{i}
\end{array}\right]\left[\begin{array}{cc}
1 & 0 \\
0 & x_{n+1}
\end{array}\right]\left[\begin{array}{l}
1 \\
1
\end{array}\right] .
$$

Now, if $M(c)$ denotes the quasi-simple regular module associated with string $c$, then it follows from Lemma 7.1 that $L_{c}$ is the cluster character corresponding to the module $M(c)$ and thus is a cluster variable in the cluster algebra $\mathcal{A}(Q, \emptyset)$.

8.3. Example for the $n$-Kronecker quiver with principal coefficients. Let $n \geq 2$ and $K_{n}^{\mathrm{pp}}$ be the quiver

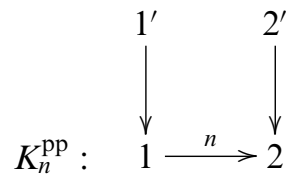

where $1 \stackrel{n}{\rightarrow} 2$ means that there are $n$ arrows from 1 to 2 . We set $F=\left\{1^{\prime}, 2^{\prime}\right\}$ so that $K_{n}^{\text {pp }}$ is the principal extension of the $n$-Kronecker quiver $K_{n}$. We fix two arrows among the $n$ arrows going from 1 to 2 , which we denote by $\alpha$ and $\beta$.

For any $p \geq 1$, we define a string representation $M^{p}$ of $K_{n}$ by setting $M^{p}(1)=\mathbf{k}^{p}$, $M^{p}(2)=\mathbf{k}^{p+1}, M^{p}(\alpha)=1_{\mathbf{k}^{p}} \oplus 0$ and $M^{p}(\beta)=0 \oplus 1_{\mathbf{k}^{p}}$ and view $M^{p}$ as a representation of $K_{n}^{\mathrm{pp}}$, which is supported on the unfrozen part $K_{n}$. For any $p \geq 1$, the $\operatorname{string} \mathbf{s}\left(M^{p}\right)$ is $\left(\alpha^{-1} \beta\right)^{p}$ so that it has length $2 p$.

We write $y_{1}=x_{1^{\prime}}$ and $y_{2}=x_{2^{\prime}}$. Then a direct computation shows that

$$
L_{M^{p}}=\frac{1}{x_{1}^{p} x_{2}^{p+1}}[1, \quad 1]\left[\begin{array}{cc}
1 & 0 \\
0 & y_{2} x_{1}^{n-1}
\end{array}\right]\left[\begin{array}{cc}
y_{1}+x_{2}^{n} & y_{1} y_{2} x_{1}^{n-1} \\
y_{1} x_{1} & y_{1} y_{2} x_{1}^{n}
\end{array}\right]^{p}\left[\begin{array}{c}
1 \\
x_{1}
\end{array}\right] .
$$

Applying Corollary 2.10, we see that there exists a realisable quadruple $\left(K_{n}^{\mathrm{pp}},\left(K_{n}\right)_{0}^{\prime}, \mathcal{C}, T\right)$. As $K_{n}^{\mathrm{pp}}$ is acyclic, it follows from [38] that $B_{T}$ is hereditary and thus Lemma 7.1 implies that the normalising vector vanishes. Thus, $X_{M^{p}}^{T}=L_{M^{p}}$ is given by formula (8.7) for every $p \geq 1$. 
8.4. Cyclic cluster-tilted algebras of Dynkin type $\mathbb{D}$. Let $B$ be the quotient of the path algebra of the quiver

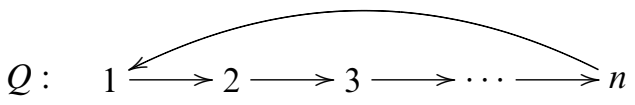

by an ideal generated by all paths of length $n-1$. Here we suppose that $n \geq 3$. $B$ is a cluster-tilted algebra of type $\mathbb{D}_{n}$ if $n \geq 4$ and a cluster-tilted algebra of type $\mathbb{A}_{3}$ if $n=3$. Let $M$ be the indecomposable module given by the Loewy series $\begin{gathered}2 \\ 3 \\ \vdots \\ m\end{gathered}$, where $2 \leq m \leq n$.

Proposition 8.1. Let $Q$ denote the above quiver and $(\mathcal{Q}, F, \mathcal{C}, T)$ be a realisable quadruple with unfrozen part $Q$. For any $i \in Q_{0}$, let, as in Section 4.1,

$$
y_{i}=\prod_{\alpha \in \mathcal{Q}_{1}(F, i)} x_{s(\alpha)} \quad \text { and } \quad z_{i}=\prod_{\alpha \in \mathcal{Q}_{1}(i, F)} x_{t(\alpha)}
$$

Let $M$ be as above. Then

$$
L_{M}=\sum_{\ell=1}^{m} \frac{x_{1} x_{2} \cdots x_{m+1}}{x_{\ell} x_{\ell+1}}\left(\prod_{i=2}^{\ell} y_{i}\right)\left(\prod_{i=\ell+1}^{m} z_{i}\right) \frac{1}{\prod_{j=2}^{m} x_{j}}
$$

if $m<n$; and if $n=m$, then $L_{M}$ is given by (8.8) divided by $x_{1}$.

Proof. We proceed by induction on $m$. If $m=2$, then $M$ is simple and $L_{M}$ is given by the exchange relation

$$
L_{M}=\left(x_{1} y_{2}+x_{3} z_{2}\right) \frac{1}{x_{2}} .
$$

On the other hand, (8.8) gives

$$
\left(\frac{x_{1} x_{2} x_{3}}{x_{1} x_{2}} z_{2}+\frac{x_{1} x_{2} x_{3}}{x_{2} x_{3}} y_{2}\right) \frac{1}{x_{2}}
$$

which is equal to the right-hand side of equation (8.9).

Now suppose $m>2$. Let $N_{M}$ be the numerator of the Laurent polynomial $L_{M}$. Our formula gives $N_{M}$ as the product of $2 m-1$ matrices:

$$
\left[\begin{array}{ll}
1 & x_{1}
\end{array}\right]\left[\begin{array}{cc}
z_{2} & 0 \\
0 & y_{2}
\end{array}\right] \cdots\left[\begin{array}{cc}
x_{m} & 0 \\
1 & x_{m-1}
\end{array}\right]\left[\begin{array}{cc}
z_{m} & 0 \\
0 & y_{m}
\end{array}\right]\left[\begin{array}{c}
x_{m+1} \\
1
\end{array}\right] .
$$

The product of the last three matrices in (8.10) is equal to

$$
\left[\begin{array}{c}
x_{m} x_{m+1} z_{m} \\
x_{m+1} z_{m}+x_{m-1} y_{m}
\end{array}\right]=\left[\begin{array}{c}
x_{m} \\
1
\end{array}\right] x_{m+1} z_{m}+\left[\begin{array}{l}
0 \\
1
\end{array}\right] x_{m-1} y_{m} .
$$


Let us denote the product of the first $2 m-4$ matrices by [ $\left.\begin{array}{ll}a & b\end{array}\right]$, so

$$
N_{M}=\left[\begin{array}{ll}
a & b
\end{array}\right]\left(\left[\begin{array}{c}
x_{m} \\
1
\end{array}\right] x_{m+1} z_{m}+\left[\begin{array}{l}
0 \\
1
\end{array}\right] x_{m-1} y_{m}\right) .
$$

Now let $M^{\prime}$ be the indecomposable module given by the Loewy series $\begin{gathered}2 \\ 3 \\ :\end{gathered}$. Then our formula gives $N_{M^{\prime}}$ as a product of $2 m-3$ matrices, and the first $2 m-4$ matrices are just the same as the first $2 m-4$ matrices in (8.10), and therefore

$$
N_{M^{\prime}}=\left[\begin{array}{ll}
a & b
\end{array}\right]\left[\begin{array}{c}
x_{m} \\
1
\end{array}\right] \text {. }
$$

Thus,

$$
N_{M}=N_{M^{\prime}} x_{m+1} z_{m}+\left[\begin{array}{ll}
a & b
\end{array}\right]\left[\begin{array}{l}
0 \\
1
\end{array}\right] x_{m-1} y_{m}
$$

which, by induction, is equal to

$$
\sum_{\ell=1}^{m-1} \frac{x_{1} x_{2} \cdots x_{m}}{x_{\ell} x_{\ell+1}}\left(\prod_{i=2}^{\ell} y_{i}\right)\left(\prod_{i=\ell+1}^{m-1} z_{i}\right) x_{m+1} z_{m}+\frac{x_{1} x_{2} \cdots x_{m}}{x_{m-1} x_{m}}\left(\prod_{i=2}^{m-1} y_{i}\right) x_{m-1} y_{m},
$$

which in turn is equal to

$$
\sum_{\ell=1}^{m-1} \frac{x_{1} x_{2} \cdots x_{m+1}}{x_{\ell} x_{\ell+1}}\left(\prod_{i=2}^{\ell} y_{i}\right)\left(\prod_{i=\ell+1}^{m} z_{i}\right)+\frac{x_{1} x_{2} \cdots x_{m+1}}{x_{m} x_{m+1}}\left(\prod_{i=2}^{m} y_{i}\right),
$$

and this shows the formula (8.8). The statement for $m=n$ follows from the normalising factor.

ACKNOWLEDGMENTS. Work on this problem was started during the 2010 South American Meeting on Representations of Algebras and Related Topics; the authors wish to thank the organisers for their kind invitation.

The first author gratefully acknowledges partial support from the NSERC of Canada, the FQRNT of Québec and the Universite de Sherbrooke.

This paper was written at the time the second author was at the Universite de Sherbrooke as a CRM-ISM postdoctoral fellow under the supervision of the first author, Thomas Brüstle and Virginie Charette.

The third author is supported by the NSF grants DMS-0908765 and DMS1001637 and by the University of Connecticut.

The fourth author gratefully acknowledges support from the NSERC of Canada and Bishop's University. 


\section{REFERENCES}

1. C. Amiot, Cluster categories for algebras of global dimension 2 and quivers with potential, Ann. Inst. Fourier (Grenoble) 59(6) (2009), 2525-2590.

2. I. Assem, T. Brüstle, G. Charbonneau-Jodoin and P. Plamondon, Gentle algebras arising from surface triangulations, Algebra Number Theory 4(2) (2010), 201-229.

3. I. Assem and G. Dupont, Friezes and a construction of the euclidean cluster variables, J. Pure and Appl. Algebra 215(10) (2011), 2322-2340.

4. I. Assem, C. Reutenauer and D. Smith, Friezes, Adv. Math. 225 (2010), 3134-3165.

5. I. Assem, D. Simson and A. Skowroński, Elements of representation theory of associative algebras, vol. 1: Techniques of representation theory (London Mathematical Society Student Texts, vol. 65) (Cambridge University Press, Cambridge, UK, 2005).

6. M. Auslander, I. Reiten and S. Smalø, Cambridge studies in advanced mathematics: Vol 36, Representation theory of Artin algebras (Cambridge University Press, Cambridge, UK, 1997). Corrected reprint of the 1995 original.

7. K. Baur and R. Marsh, Categorification of a frieze pattern determinant. arXiv:1008.5329v1 [math.CO], 2010.

8. A. Buan, O. Iyama, I. Reiten and J. Scott, Cluster structures for 2-Calabi-Yau categories and unipotent groups, Compos. Math. 145(4) (2009), 1035-1079.

9. A. Buan, O. Iyama, I. Reiten and D. Smith, Mutation of cluster-tilting objects and potentials. To appear in American J. Math. arXiv:0804.3813v3 [math.RT], 2009.

10. A. Buan, R. Marsh, M. Reineke, I. Reiten and G. Todorov, Tilting theory and cluster combinatorics, Adv. Math. 204(2) (2006), 572-618.

11. A. Buan, R. Marsh and I. Reiten, Cluster-tilted algebras, Trans. Amer. Math. Soc. 359(1) (2007), 323-332.

12. A. Buan, R. Marsh and I. Reiten, Denominators of cluster variables, J. Lond. Math. Soc. (2) 79(3) (2009), 589-611.

13. M. Butler and C. M. Ringel, Auslander-Reiten sequences with few middle terms and applications to string algebras, Commun. Algebra, 15(1-2) (1987), 145-179.

14. P. Caldero and F. Chapoton, Cluster algebras as Hall algebras of quiver representations, Commentarii Mathematici Helvetici 81 (2006), 596-616.

15. P. Caldero, F. Chapoton and R. Schiffler, Quivers with relations arising from clusters ( $A_{n}$ case), Trans. AMS 358 (2006), 1347-1354.

16. P. Caldero and B. Keller, From triangulated categories to cluster algebras II, Annales Scientifiques de l'Ecole Normale Supérieure, 39(4) (2006), 83-100.

17. G. Caroll and G. Price, The new combinatorial models for the Ptolemy recurrence. unpublished.

18. G. Cerulli Irelli, Quiver Grassmannians associated with string modules, J. Algebr. Comb. 33(2) (2011), 259-276.

19. J. Conway and H. Coxeter, Triangulated polygons and frieze patterns, Math. Gaz. 57(401) (1973), 175-183.

20. J. Conway and H. Coxeter, Triangulated polygons and frieze patterns, Math. Gaz. 57(400) (1973), 87-94.

21. H. Coxeter, Frieze patterns, Acta Arith. 18 (1971), 297-310.

22. H. Derksen, J. Weyman and A. Zelevinsky, Quivers with potentials and their representations. I. Mutations, Selecta Math. (N.S.) 14(1) (2008), 59-119.

23. G. Dupont, Positivity in coefficient-free rank two cluster algebras, Electron. J. Comb. 16(1) (2009), R98.

24. G. Dupont, Caldero-Keller approach to the denominators of cluster variables, Commun. Algebra 38(7) (2010), 2538-2549.

25. G. Dupont, Cluster multiplication in regular components via generalized Chebyshev polynomials, Algebr. Represent. Theory. To appear.

26. G. Dupont, Quantized Chebyshev polynomials and cluster characters with coefficients, J. Algebr. Comb. 31(4) (2010), 501-532.

27. S. Fomin, M. Shapiro and D. Thurston, Cluster algebras and triangulated surfaces. I. Cluster complexes, Acta Math. 201(1) (2008), 83-146.

28. S. Fomin and A. Zelevinsky, Cluster algebras I: Foundations, J. Amer. Math. Soc. 15 (2002), 497-529. 
29. S. Fomin and A. Zelevinsky, Cluster algebras IV: Coefficients, Compos. Math. 143(1) (2007), 112-164.

30. A. Fordy and R. Marsh, Cluster mutation-periodic quivers and associated Laurent sequences, J. Algebr. Comb. 34(1) (2011), 19-66.

31. C. Fu and B. Keller, On cluster algebras with coefficients and 2-Calabi-Yau categories, Trans. Amer. Math. Soc. 362 (2010), 859-895.

32. $\mathrm{C}$. Fu and $\mathrm{P}$. Liu, Lifting to cluster-tilting objects in 2-Calabi-Yau triangulated categories, Comm. Algebra 37(7) (2009), 2410-2418.

33. P. Gabriel, Auslander-Reiten sequences and representation-finite algebras, In Representation theory, I (Proc. Workshop, Carleton Univ., Ottawa, Ont., 1979), vol. 831 of Lecture Notes in Math. (Springer, Berlin, 1980), 1-71.

34. N. Haupt, Euler characteristics of quiver Grassmannians and Ringel-Hall algebras of string algebras, Algebra. Represent. Theory. To appear.

35. B. Keller, On triangulated orbit categories, Doc. Math. 10 (2005), 551-581.

36. B. Keller and M. Van den Bergh, Deformed Calabi-Yau completions, J. reine angew. Math. 2011(654) (2011), 125-180.

37. B. Keller and I. Reiten, Cluster-tilted algebras are Gorenstein and stably Calabi-Yau, Adv. Math. 211(1) (2007), 123-151.

38. B. Keller and I. Reiten, Acyclic Calabi-Yau categories, Compos. Math. 144(5) (2008), $1332-1348$.

39. B. Keller and S. Scherotzke, Linear recurrence relations for cluster variables of affine quivers. arXiv:1004.0613v2 [math.RT], 2010.

40. D. Labardini-Fragoso, Quivers with potentials associated to triangulated surfaces, Proc. London Math. Soc. 98(3) (2009), 787-839.

41. S. Morier-Genoud, V. Osvienko and S. Tabachnikov, 2-frieze patterns and the cluster structure of the space of polygons. arXiv:1008.3359v2 [math.AG], 2010.

42. G. Musiker, A graph theoretic expansion formula for cluster algebras of classical type, Ann. Comb. 15(1) (2011), 147-184.

43. G. Musiker and J. Propp, Combinatorial interpretations for rank-two cluster algebras of affine type, Electron. J. Combin. 14(1) (2007), Research Paper 15, 23 pp. (electronic).

44. G. Musiker, R. Schiffler and L. Williams, Positivity for cluster algebras from surfaces, Adv. Math. doi:10.1016/j.aim.2011.04.018.

45. H. Nakajima, Quiver varieties and cluster algebras, Kyoto J. Math. 51(1) (2011), 71-126.

46. Y. Palu, Cluster characters for 2-Calabi-Yau triangulated categories, Ann. Inst. Fourier (Grenoble) 58(6) (2008), 2221-2248.

47. P. Plamondon, Cluster algebras via cluster categories with infinite-dimensional morphism spaces. arXiv:1004.0830v2 [math.RT], 2010.

48. J. Propp, The combinatorics of frieze patterns and Markoff numbers. arXiv:math/0511633v4 [math.CO], 2008. 2010.

49. F. Qin, Quantum cluster variables via Serre polynomials. arXiv:1004.4171v2 [math.QA],

50. I. Reiten, Calabi-Yau categories. Talk at the meeting: Calabi-Yau algebras and NKoszul algebras (CIRM), 2007.

51. R. Schiffler, On cluster algebras arising from unpunctured surfaces II, Adv. Math. 223 (2010), 1885-1923.

52. R. Schiffler and H. Thomas, On cluster algebras arising from unpunctured surfaces, Int. Math. Res. Not. 17 (2009), 3160-3189.

53. P. Sherman and A. Zelevinsky, Positivity and canonical bases in rank 2 cluster algebras of finite and affine types, Mosc. Math. J. 4 (2004), 947-974.

54. D. Smith, On tilting modules over cluster-tilted algebras, Illinois J. Math. 52 (2008), $1223-1247$.

55. B. Wald and J. Waschbüsch, Tame biserial algebras, J. Algebra 95(2) (1985), 480-500. 